UNITU-THEP-26/2002

http://xxx.lanl.gov/abs/hep-ph/0301094

\title{
Non-perturbative Propagators, Running Coupling and Dynamical Quark Mass of Landau gauge QCD
}

\author{
C. S. Fischer* and R. Alkofer ${ }^{\dagger}$ \\ Institute for Theoretical Physics, University of Tübingen \\ Auf der Morgenstelle 14, D-72076 Tübingen, Germany
}

(Dated: October 22, 2018)

\begin{abstract}
The coupled system of renormalized Dyson-Schwinger equations for the quark, gluon and ghost propagators of Landau gauge QCD is solved within truncation schemes. These employ bare as well as non-perturbative ansätze for the vertices such that the running coupling as well as the quark mass function are independent of the renormalization point. The one-loop anomalous dimensions of all propagators are reproduced. Dynamical chiral symmetry breaking is found, the dynamically generated quark mass agrees well with phenomenological values and corresponding results from lattice calculations. The effects of unquenching the system are small. In particular the infrared behavior of the ghost and gluon dressing functions found in previous studies is almost unchanged as long as the number of light flavors is smaller than four.
\end{abstract}

Keywords: Confinement, dynamical chiral symmetry breaking, strong QCD, running coupling, gluon propagator, quark propagator, Dyson-Schwinger equations, Infrared behavior

PACS: 12.38.Aw 14.65.Bt 14.70.Dj 12.38.Lg 11.30.Rd 11.15.Tk 02.30.Rz

\footnotetext{
* Supported by the European Graduate School Basel-Tuebingen

E-Mail: chfi@tphys.physik.uni-tuebingen.de

† E-Mail: reinhard.alkofer@uni-tuebingen.de
} 


\section{INTRODUCTION}

Based on many observations in hadron physics spontaneous breaking of chiral symmetry and the dynamical generation of quark masses are expected to occur in Quantum Chromo Dynamics (QCD). The precise origin of this non-perturbative phenomenon as well as its relation to quark confinement are still little understood. Further studies of these issues have to build on reliable non-perturbative methods. And, as confinement is expected to be correlated with infrared singularities, continuum-based methods will be required in addition to Monte Carlo lattice calculations. To this end we note that the Schwinger-Dyson equations (DSEs) of QCD can address directly the infrared region of momentum.

The DSEs for the propagators of QCD form a coupled system of equations. In Landau gauge these have been investigated in two lines of research. On the one hand, the DSEs of pure Yang-Mills theory have been explored with the aim to reveal the infrared behavior of the ghost and gluon propagator and their relation to gluon confinement (see e.g. the review [1] and references therein). On the other hand, the quark DSEs have been studied extensively on the purpose of model building. These models have been used in the frameworks of BetheSalpeter equations and finite temperature field theory to describe hadronic properties and reactions in a semi-phenomenological way (see e.g. the review [2] and references therein).

In this article numerical results for the coupled set of quark, gluon and ghost propagators, including the backreaction of the quarks on the ghosts and gluons, are presented for the first time. In the quark DSE we will study the mechanism of dynamical chiral symmetry breaking by which physical quark masses are generated even though the bare quark masses in the Lagrangian are zero. This is a genuine non-perturbative effect as it is well known that for vanishing bare masses the renormalized masses remain zero at each order in perturbation theory. In addition to the phenomenon of mass generation we are interested in quark confinement. Single quark states have non-vanishing color charge and are therefore not contained in the physical part of the state space of QCD. This physical subspace supports a positive (semi-)definite metric whereas the remaining state space of QCD contains negative norm states as well. Consequently, negative norm contributions to the quark propagator would provide evidence for quark confinement.

This paper is organized as follows: In the next section suitable ansätze for the quarkgluon vertex will be constructed such that the DSE for the quark propagator guarantees the realization of two important consistency conditions. These are (i) the independence of the dynamically generated quark mass function from the renormalization point and (ii) the correct asymptotic behavior at large momenta such that the anomalous dimensions of dressing and mass functions are correct in one-loop order. Fortunately, the corresponding DSEs for the fermions of QED are well studied (a short overview is given e.g. in [3]). We will dwell on these results and construct non-Abelian generalizations of Abelian vertices, which have the desired properties.

In the following section we present solutions for the quenched system of quark, ghost and gluon DSEs, i.e. we neglect the quark-loop in the gluon equation. The Yang-Mills sector is hereby treated in a truncation scheme for the ghost and gluon equations that already has been employed in ref. [4]. This scheme improves on older ones [5, 6], provides an explicit numerical solution for the infrared analysis given in refs. 7, 8], and its results are in almost quantitative agreement with corresponding results of lattice calculations for the gluon and ghost propagators [9, 10, 11, 12]. The main purpose of calculating the quark propagator in quenched approximation is to allow for a comparison with corresponding recent lattice results [13, 14]. We find very good agreement of our results for the quark renormalization 
function and the momentum-dependent dynamical quark mass with the lattice results if the quark-gluon vertex functions constructed in Sect. 2 are employed. We then proceed to the unquenched case and incorporate the quark-loop into our truncation scheme for the ghost and gluon DSE. We present solutions for the full coupled system of DSEs for the quark, ghost and gluon propagators. Compared to the quenched case we will find only moderate differences for the number of light flavors $N_{f} \leq 3$. Whereas we are able to demonstrate positivity violation in the gluon propagator (and thus gluon confinement) we have not been able to draw definite conclusions on the (non-)positivity of the quark propagator. In the last section we summarize our results and present some conclusions.

\section{THE QUARK DYSON-SCHWINGER EQUATION}

The coupled DSEs for the gluon, ghost and quark propagators in Landau gauge QCD are shown in in fig. 1. As stated in the introduction we will employ first the quenched approximation, i.e. we will neglect the quark-loop in the gluon equation. This will allow us to assess the quality of our quark DSE solution by comparing to lattice calculations of the quark propagator [13] performed so far only in quenched approximation. As we furthermore employ the gluon propagator obtained in ref. [4] the unknown element to be determined in the quark DSE is the dressed quark-gluon vertex.

Following the conventions and notations of ref. [1] the renormalized quark DSE with appropriate quark wave function and quark-gluon vertex renormalization constants, $Z_{2}$ and $Z_{1 F}$, respectively, reads

$$
S^{-1}(p)=Z_{2} S_{0}^{-1}(p)+g^{2} Z_{1 F} C_{F} \int \frac{d^{4} q}{16 \pi^{4}} \gamma_{\mu} S(q) \Gamma_{\nu}(q, q-p) D_{\mu \nu}(q-p) .
$$

The factor $C_{F}=\left(N_{c}^{2}-1\right) / 2 N_{c}$ in front of the integral stems from the color trace of the loop. The symbol $\Gamma_{\nu}(q, q-p)$ denotes the full quark-gluon vertex. Suppressing color indices the quark and gluon propagators in Landau gauge are given by

$$
\begin{aligned}
S(p) & =\frac{1}{-i p A\left(p^{2}\right)+B\left(p^{2}\right)}=A^{-1}\left(p^{2}\right) \frac{i p+M\left(p^{2}\right)}{p^{2}+M^{2}\left(p^{2}\right)}, \\
S_{0}(p) & =\frac{1}{-i p+m_{0}}, \\
D_{\mu \nu}(p) & =\left(\delta_{\mu \nu}-\frac{p_{\mu} p_{\nu}}{p^{2}}\right) \frac{Z\left(p^{2}\right)}{p^{2}}
\end{aligned}
$$

where the quark mass function $M$ is defined as $M\left(p^{2}\right)=B\left(p^{2}\right) / A\left(p^{2}\right)$.

The inverse of the vector self-energy, $1 / A\left(q^{2}\right)$, is often denoted as the 'quark wave function renormalization'. The bare quark propagator $S_{0}\left(p^{2}\right)$ contains the unrenormalized quark mass $m_{0}\left(\Lambda^{2}\right)$ which depends on the cutoff $\Lambda$ of the theory. The bare mass is related to the renormalized mass $m_{R}\left(\mu^{2}\right)$ via the renormalization constant $Z_{m}$ :

$$
m_{0}\left(\Lambda^{2}\right)=Z_{m}\left(\mu^{2}, \Lambda^{2}\right) m_{R}\left(\mu^{2}\right) .
$$



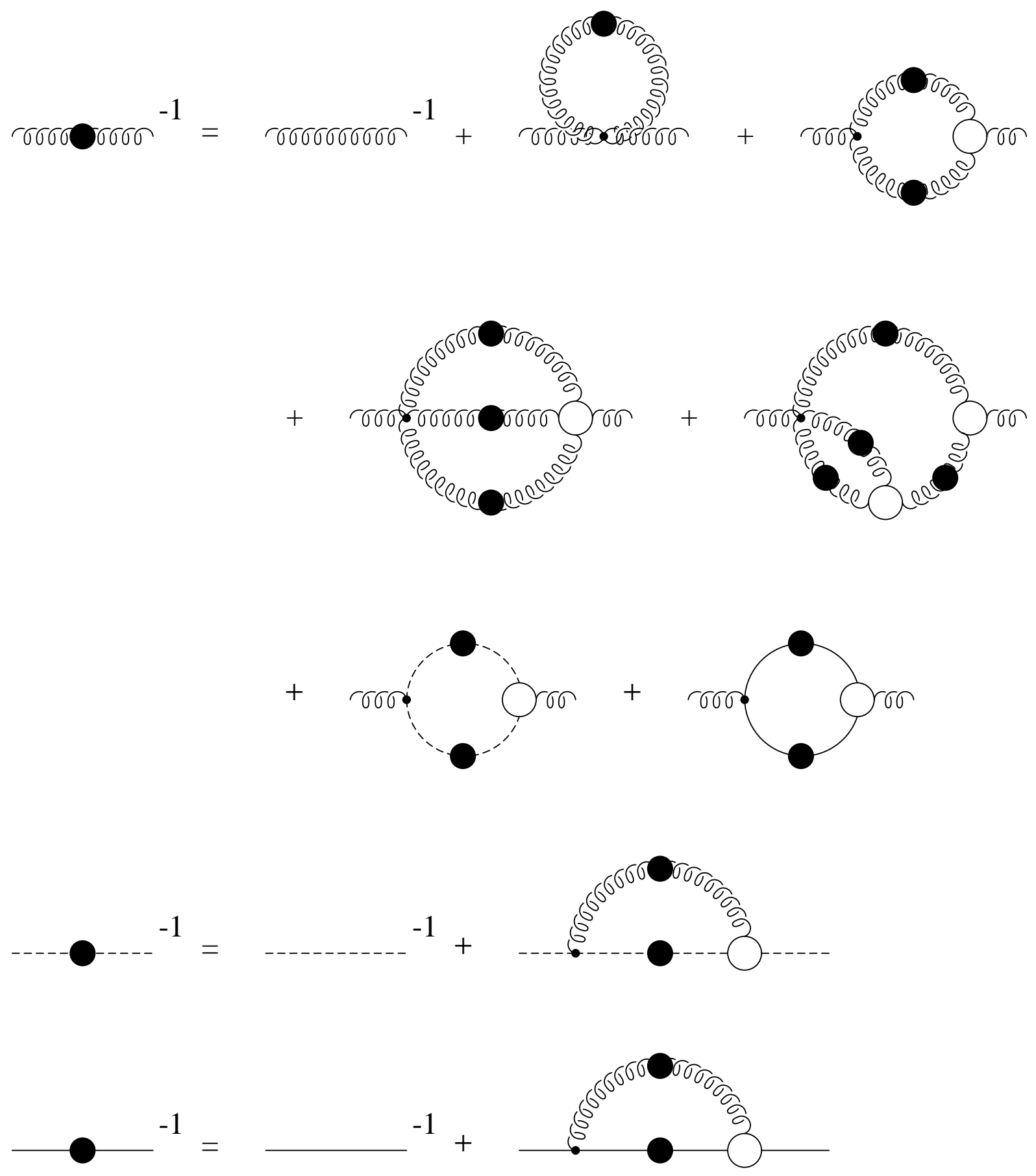

FIG. 1: Diagrammatic representation of the Dyson-Schwinger equations for the gluon, ghost and quark propagators. The wiggly, dashed and solid lines represent the propagation of gluons, ghosts and quarks, respectively. A filled blob represents a full propagator and a circle indicates a oneparticle irreducible vertex. 
Here $\mu^{2}$ is the (squared) renormalization scale. Note that the propagator functions $A, B$ and $Z$ depend on this renormalization scale. Therefore in the following we will use the notation $A\left(p^{2}, \mu^{2}\right), B\left(p^{2}, \mu^{2}\right)$ and $Z\left(p^{2}, \mu^{2}\right)$.

The renormalized and the bare vector self-energy, $A$ and $A_{0}$, are related by

$$
A_{0}^{-1}\left(p^{2}, \Lambda^{2}\right)=Z_{2}\left(\mu^{2}, \Lambda^{2}\right) A^{-1}\left(p^{2}, \mu^{2}\right)
$$

In Landau gauge the loop corrections to the vector self energy are finite. Correspondingly $Z_{2}\left(\mu^{2}, \Lambda^{2}\right)$ stays finite when the cutoff is sent to infinity, and we have $0<Z_{2}\left(\mu^{2}, \Lambda^{2}\right)<1$. Furthermore, in Landau gauge the ghost-gluon vertex is not ultraviolet divergent, and we can choose $\tilde{Z}_{1}=1$ [15]. The Slavnov-Taylor identity for the quark-gluon vertex renormalization factor $Z_{1 F}$ thus simplifies [16],

$$
Z_{1 F}=\frac{\tilde{Z}_{1} Z_{2}}{\tilde{Z}_{3}}=\frac{Z_{2}}{\tilde{Z}_{3}} .
$$

Previous studies of the quark DSE in the so-called Abelian approximation (see e.g. the review [2] or the recent summary [17] and references therein) as well as the recent investigation in ref. [18] assume implicit cancellations between the full quark-gluon vertex and the dressed gluon propagator in the integral over the kernel of the quark DSE. Furthermore, in the tensor structure of the quark-gluon vertex only a term proportional to $\gamma_{\mu}$ is employed.

In the current investigation we do not have to rely on implicit cancellations since we calculated explicit solutions for the dressed gluon and ghost propagators [4]. We will also construct explicit non-perturbative ansätze for the quark-gluon vertex. We note that very recently lattice results for the quark-gluon vertex became available [19]. However, at present the error bars from such simulations are too large to use the lattice results as guideline in the construction of reliable ansätze for the quark-gluon vertex. To proceed we assume that one may approximately factorize the quark-gluon vertex

$$
\Gamma_{\nu}(q, k)=V_{\nu}^{a b e l}(p, q, k) W^{\neg a b e l}\left(p^{2}, q^{2}, k^{2}\right),
$$

with $p$ and $q$ denoting the quark momenta and $k=(q-p)$ the gluon momentum. The nonAbelian factor $W^{\neg a b e l}$ multiplies an Abelian part $V_{\nu}^{\text {abel }}$, which carries the tensor structure of the vertex. This ansatz is motivated by the aim to respect gauge invariance as much as possible on the present level of truncation.

The Slavnov-Taylor identity (STI) for the quark-gluon vertex is given by [16]

$$
G^{-1}\left(k^{2}\right) i k_{\mu} \Gamma_{\mu}(q, k)=S^{-1}(p) H(q, p)-H(q, p) S^{-1}(q),
$$

with $G\left(k^{2}\right)$ being the ghost dressing function and $H(q, p)$ the ghost-quark scattering kernel. At present the non-perturbative behavior of the ghost-quark scattering kernel is unknown. Therefore we cannot solve this STI explicitly. However, comparing the structure of eq. (9) with the corresponding Ward identity of QED,

$$
i k_{\mu} \Gamma_{\mu}^{Q E D}(q, k)=S^{-1}(p)-S^{-1}(q),
$$

we are able to infer some information: Whereas the ghost fields of QED decouple from the theory and consequently do not show up in the Ward identity there is an explicit factor of $G^{-1}\left(k^{2}\right)$ on the left hand side of eq. (9). We therefore suspect the quark-gluon vertex of QCD to contain an additional factor of $G\left(k^{2}\right)$ compared to the fermion-photon vertex of QED. Some additional ghost dependent structure seems necessary to account for the ghost-quark 
scattering kernel on the right hand side of eq. (9). For simplicity we assume the whole ghost dependence of the vertex to be contained in a non-Abelian factor multiplying an Abelian tensor structure. For quenched calculations and in the context of angular approximated DSEs a similar strategy has already been adopted in refs. [20, 21]. The Abelian part of the vertex, $V_{\nu}^{\text {abel }}$, will be adopted in the following from analog ansätze in QED. The Ward identity (10) has been solved e.g. in ref. [22] such that kinematical singularities are avoided. Furthermore transverse parts of the fermion-photon vertex have been fixed such that multiplicative renormalizability in the Abelian fermion DSE is satisfied for all linear covariant gauges [23]. The resulting vertex is known as the Curtis-Pennington (CP) vertex.

The non-Abelian factor $W^{\neg a b e l}$ is chosen such that the resulting quark propagator fulfills two conditions:

(i) The quark mass function $M\left(p^{2}\right)$ should be independent of the renormalization point $\mu^{2}$.

(ii) The anomalous dimension $\gamma_{m}$ of the mass function known from perturbation theory should be recovered in the ultraviolet.

In the course of this section we will prove the vertex ansatz

$$
\begin{aligned}
W^{\neg a b e l}\left(p^{2}, q^{2}, k^{2}\right)= & G^{2}\left(k^{2}, \mu^{2}\right) \tilde{Z}_{3}\left(\mu^{2}, \Lambda^{2}\right) \frac{\left(G\left(k^{2}, \mu^{2}\right) \tilde{Z}_{3}\left(\mu^{2}, \Lambda^{2}\right)\right)^{-2 d-d / \delta}}{\left(Z\left(k^{2}, \mu^{2}\right) Z_{3}\left(\mu^{2}, \Lambda^{2}\right)\right)^{d}} \\
V_{\nu}^{a b e l}(p, q, k)= & \Gamma_{\nu}^{C P}(p, q, k) \\
= & \frac{A\left(p^{2}, \mu^{2}\right)+A\left(q^{2}, \mu^{2}\right)}{2} \gamma_{\nu}+i \frac{B\left(p^{2}, \mu^{2}\right)-B\left(q^{2}, \mu^{2}\right)}{p^{2}-q^{2}}(p+q)_{\nu} \\
& +\frac{A\left(p^{2}, \mu^{2}\right)-A\left(q^{2}, \mu^{2}\right)}{2\left(p^{2}-q^{2}\right)}(\not p+\not q)(p+q)_{\nu} \\
& +\frac{A\left(p^{2}, \mu^{2}\right)-A\left(q^{2}, \mu^{2}\right)}{2}\left[\left(p^{2}-q^{2}\right) \gamma_{\nu}-(p-\not q)(p+q)_{\nu}\right] \\
& \times \frac{p^{2}+q^{2}}{\left(p^{2}-q^{2}\right)^{2}+\left(M^{2}\left(p^{2}\right)+M^{2}\left(q^{2}\right)\right)^{2}}
\end{aligned}
$$

with the new parameter $d$ to satisfy the conditions (i) and (ii). Again $k$ denotes the gluon momentum and $p$ and $q$ the quark momenta. The anomalous dimension $\delta$ of the ghost propagator is $\delta=-9 N_{c} /\left(44 N_{c}-8 N_{f}\right)$, i.e. the corresponding value at one loop order for $N_{c}$ colors and $N_{f}$ flavors. The Abelian part of the vertex is given by the Curtis-Pennington (CP) vertex $\Gamma_{\nu}^{C P}(p, q, k)$.

From a systematic point of view the newly introduced parameter $d$ in the non-Abelian part of the vertex is completely arbitrary. Our numerical results, however, will indicate that values around the somewhat natural choice $d=0$ match best with lattice simulations, see below.

For comparison we will also employ the much simpler vertex $\Gamma_{\nu}=V_{\nu}^{\text {abel }} W^{\neg a b e l}$ with $W^{\neg a b e l}$ given by eq. (11) and

$$
V_{\nu}^{a b e l}(p, q, k)=Z_{2}\left(\mu^{2}, \Lambda^{2}\right) \gamma_{\nu}
$$

where we have taken the bare Abelian vertex, $\gamma_{\nu}$, multiplied with an extra factor of $Z_{2}$. In Landau gauge this construction also satisfies the conditions (i) and (ii), as will be shown 
in the next two subsections. Furthermore in the numerical treatment we will additionally employ a vertex where the last, transverse term in Eq. (13) is left out, i.e. a generalized Ball-Chiu (BC) vertex. In Landau gauge such a vertex also satisfies the conditions (i) and (ii).

\section{Multiplicative renormalizability of the quark equation}

To proceed we first substitute the simpler vertex ansatz (14) into the quark equation (11). By taking the Dirac trace once with and once without multiplying the equation with $\not p$ we project out the mass function $M\left(p^{2}\right)$ and the vector self energy $A\left(p^{2}\right)$. To ease notation we will use in the following the abbreviations $x=p^{2}, y=q^{2}$ and $z=(p-q)^{2}$ for the squared momenta, also $s=\mu^{2}$ for the squared renormalization point and $L=\Lambda^{2}$ for the squared cutoff of the theory. We arrive at

$$
\begin{gathered}
M(x) A(x, s)=Z_{2}(s, L) m_{0}(L)+\frac{Z_{2}(s, L)}{3 \pi^{3}} \int d^{4} q\left\{\frac{\alpha(z)}{z\left(y+M^{2}(y)\right)} Z_{2}(s, L) A^{-1}(y, s) \times\right. \\
\left.\frac{\left(G(z, s) \tilde{Z}_{3}(s, L)\right)^{-2 d-d / \delta}}{\left(Z(z, s) Z_{3}(s, L)\right)^{d}} 3 M(y)\right\}, \\
\begin{array}{c}
A(x, s)=Z_{2}(s, L)+\frac{Z_{2}(s, L)}{3 \pi^{3}} \int d^{4} q\left\{\frac{\alpha(z)}{x z\left(y+M^{2}(y)\right)} Z_{2}(s, L) A^{-1}(y, s) \times\right. \\
\left.\left(G(z, s) \tilde{Z}_{3}(s, L)\right)^{-2 d-d / \delta}\left(-z+\frac{x+y}{2}+\frac{(x-y)^{2}}{2 z}\right)\right\},
\end{array}
\end{gathered}
$$

where we have used the following expression for the running coupling $\alpha$ in Landau gauge [5]

$$
\alpha(x)=\frac{g^{2}}{4 \pi} Z(x, s) G^{2}(x, s)=\alpha(s) Z(x, s) G^{2}(x, s) .
$$

From eqs. (15) and (16) it is clear that the choice of the parameter $d=0$ is special since then the only input from the Yang-Mills sector is the running coupling.

The behavior of eqs. (15), (16) under renormalization can be explored by changing the renormalization point $s=\mu^{2}$ to a new point $t=\nu^{2}$. We first note that the factor stemming from the non-Abelian part of the quark-gluon vertex is not affected by such a change:

$$
\frac{\left(G(z, s) \tilde{Z}_{3}(s, L)\right)^{-2 d-d / \delta}}{\left(Z(z, s) Z_{3}(s, L)\right)^{d}}=\frac{\left(G(z, t) \tilde{Z}_{3}(t, L)\right)^{-2 d-d / \delta}}{\left(Z(z, t) Z_{3}(t, L)\right)^{d}} .
$$

This can be seen easily with the help of the relations

$$
\begin{aligned}
G_{0}(x, L) & =G(x, s) \tilde{Z}_{3}(s, L), \\
Z_{0}(x, L) & =Z(x, s) Z_{3}(s, L),
\end{aligned}
$$

between the unrenormalized and renormalized ghost and gluon dressing functions. Further-

more, the running coupling $\alpha(z)$ is independent of the renormalization point, for a detailed discussion of this property using the expression (17) see ref. [5]. 


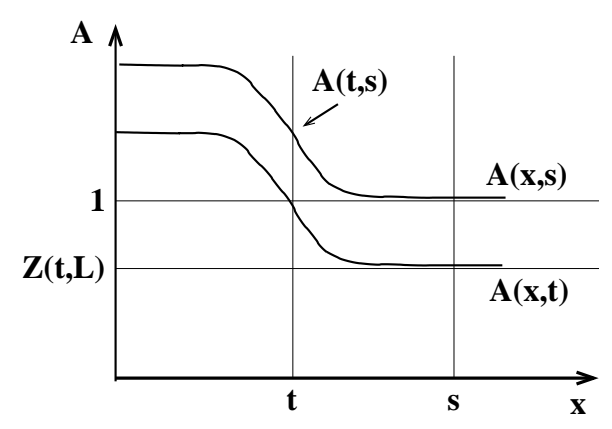

FIG. 2: Sketch of a finite renormalization from a perturbative point $s$ to a non-perturbative point $t$ for the vector self-energy $A$.

From eq. (6) we infer

$$
Z_{2}(t, L) A^{-1}(x, t)=Z_{2}(s, L) A^{-1}(x, s) .
$$

With the renormalization condition $A(t, t)=1$ we have

$$
Z_{2}(t, L)=Z_{2}(s, L) A^{-1}(t, s),
$$

and subsequently

$$
A(x, t)=A(x, s) A^{-1}(t, s) .
$$

Substituting eqs. (21) and (22) into the Dyson-Schwinger equations (15) we find the mass function $M(x)$ to be independent of the renormalization point, i.e. condition (i) is satisfied. Note that without the extra factor of $Z_{2}$ in the Abelian part of the vertex (14) we would violate this condition.

Before we examine the case of the more sophisticated Curtis-Pennington type vertex (13) two remarks are in order. First, according to perturbation theory we have $A(x \rightarrow \infty, s) \rightarrow 1$ and $Z_{2}(s, L) \rightarrow 1$ for large renormalization points $s$. However, this is just a special case of the general relation

$$
A(x \rightarrow \infty, s) \rightarrow Z_{2}(s, L)
$$

which can be inferred from eqs. (21) and (22). In Fig. 2 we sketch the vector self energy renormalized at two different points $s$ and $t$, with $s$ in the perturbative and $t$ in the nonperturbative region of momentum. Of course, this mechanism will be found again in the numerical results, see below. Second, the appearance of the ghost and gluon renormalization factors $Z_{3}(L)$ and $\tilde{Z}_{3}(L)$ in the interaction kernel of the quark equation is due to the non-abelian part of the employed quark-gluon vertex ansatz. Certainly, the renormalized functions $M(x)$ and $A(x, s)$ should not depend on the cutoff of the integral. The balance of cutoff dependent quantities in the equation is controlled by various factors of $Z_{2}(L)$ and $Z_{m}(L)$. We have to take care not to disturb this balance by the vertex ansatz. Thus the non-Abelian part of the quark-gluon vertex contains such powers of $Z_{3}(L)$ and $\tilde{Z}_{3}(L)$ that the cutoff dependence of these quantities cancel. This can be easily checked using the scaling behavior

$$
\begin{aligned}
& Z_{3}(s, L)=\left(\frac{\alpha(L)}{\alpha(s)}\right)^{\gamma} \\
& \tilde{Z}_{3}(s, L)=\left(\frac{\alpha(L)}{\alpha(s)}\right)^{\delta}
\end{aligned}
$$


of the renormalization factors for $L \rightarrow \infty$ and the relation $\gamma+2 \delta+1=0$ for the anomalous dimensions of the gluon and the ghost, respectively.

Along the same lines as for the bare vertex construction we prove condition (i) for the Curtis-Pennington type vertex. Plugging eqs. (11), (13) into the quark equation (11) and projecting onto $M(x)$ and $A(x)$ we arrive at

$$
\begin{aligned}
M(x) A(x, s)= & Z_{2}(s, L) m_{0}(L)+\frac{Z_{2}(s, L)}{3 \pi^{3}} \int d^{4} q \frac{\alpha(z)}{z\left(y+M^{2}(y)\right)} \frac{\left(G(z, s) \tilde{Z}_{3}(s, L)\right)^{-2 d-d / \delta}}{\left(Z(z, s) Z_{3}(s, L)\right)^{d}} \\
\times A^{-1}(y, s) & {\left[\frac{3}{2}(A(x, s)+A(y, s)) M(y)\right.} \\
& +\frac{1}{2}(\Delta A M(y)-\Delta B)\left(-z+2(x+y)-\frac{(x-y)^{2}}{z}\right) \\
& \left.+\frac{3}{2}(A(x, s)-A(y, s)) M(y) \Omega(x, y)(x-y)\right] \\
A(x, s)=Z_{2}(s, L)+\frac{Z_{2}(s, L)}{3 \pi^{3}} \int d^{4} q \frac{\alpha(z)}{x z\left(y+M^{2}(y)\right)} \frac{\left(G(z, s) \tilde{Z}_{3}(s, L)\right)^{-2 d-d / \delta}}{\left(Z(z, s) Z_{3}(s, L)\right)^{d}} & \\
\times A^{-1}(y, s) & \left(-z+\frac{x+y}{2}+\frac{(x-y)^{2}}{2 z}\right) \frac{A(x, s)+A(y, s)}{2} \\
- & \left(\frac{\Delta A}{2}(x+y)+\Delta B M(y)\right)\left(-\frac{z}{2}+(x+y)-\frac{(x-y)^{2}}{2 z}\right) \\
+ & \left.\frac{3}{2}(A(x, s)-A(y, s)) M(y) \Omega(x, y)\left(\frac{x^{2}-y^{2}}{2}-z^{2} \frac{x-y}{2}\right)\right] .
\end{aligned}
$$

Here we have used the abbreviations

$$
\begin{aligned}
\Delta A & =\frac{A(x, s)-A(y, s)}{x-y}, \\
\Delta B & =\frac{B(x, s)-B(y, s)}{x-y}, \\
\Omega(x, y) & =\frac{x+y}{(x-y)^{2}+\left(M^{2}(x)+M^{2}(y)\right)^{2}} .
\end{aligned}
$$

With the help of the relations (21) and (22) we find again the quark equations (25), (26) to be consistently renormalized. Employing

$$
B(x, t)=B(x, s) A^{-1}(t, s)
$$

it can be seen directly that the mass function

$$
M(x)=B(x, s) / A(x, s)
$$

is independent of the renormalization point. (The same is true for a Ball-Chiu type vertex, which is the Curtis-Pennington construction (13) without the transverse term proportional to $\Omega(x, y)$. Note that in different gauges than Landau gauge only the Curtis-Pennington construction would satisfy condition (i), similar to QED [23].) 


\section{Ultraviolet analysis of the quark equation}

In this subsection we will show that the ansätze (13) and (14) for the quark-gluon vertex both lead to the correct perturbative limit of the quark mass function $M(x)$. We first examine the case of the bare vertex construction, eq. (15).

The ghost and gluon dressing functions $G$ and $Z$ are slowly varying for large momenta according to their perturbative limit. For loop momenta $y$ larger than the external momentum $x$ we are therefore justified to employ the angular approximation $G(z), Z(z) \rightarrow G(y), Z(y)$, see ref. [4]. Furthermore, there is a region $x_{0}<y<x$ where the approximation $G(z), Z(z) \rightarrow G(x), Z(x)$ is adequate. We are then able to carry out the angular integrals in eq. (15). If we additionally take the external momentum $x$ to be large enough all masses in the denominators become negligible since the integral is dominated by loop momenta $y \approx x$. We then obtain

$$
\begin{aligned}
M(x) A(x, s)= & Z_{2}(s, L) m_{0}(L) \\
& +\frac{Z_{2}(s, L)}{\pi} \frac{\alpha(x)}{x} \int_{x_{0}}^{x} d y Z_{2}(s, L) A^{-1}(y, s) \frac{\left(G(z, s) \tilde{Z}_{3}(s, L)\right)^{-2 d-d / \delta}}{\left(Z(z, s) Z_{3}(s, L)\right)^{d}} M(y) \\
& +\frac{Z_{2}(s, L)}{\pi} \int_{x}^{L} d y \frac{\alpha(y)}{y} Z_{2}(s, L) A^{-1}(y, s) \frac{\left(G(z, s) \tilde{Z}_{3}(s, L)\right)^{-2 d-d / \delta}}{\left(Z(z, s) Z_{3}(s, L)\right)^{d}} M(y),
\end{aligned}
$$

where the integral from $y=0$ to $y=x_{0}$ has already been neglected.

For large momenta $y>x_{0}$ the wave function renormalization $A^{-1}$ and the renormalization factor $Z_{2}$ cancel each other according to eq. (23). The ultraviolet limit of the ghost and gluon dressing functions from their respective DSEs in quenched approximation has been discussed in ref. [4] and found to be in agreement with resumed perturbation theory to one-loop order. As will be seen in the discussion beyond quenched approximation below (see eqs. (89)), we also obtain the correct anomalous dimensions for the ghost and gluon dressing functions $G$ and $Z$ in the case of $N_{f} \neq 0$. We thus use the general perturbative limit

$$
\begin{aligned}
& G(z)=G(s)\left[\omega \log \left(\frac{z}{s}\right)+1\right]^{\delta}, \\
& Z(z)=Z(s)\left[\omega \log \left(\frac{z}{s}\right)+1\right]^{\gamma},
\end{aligned}
$$

with $\omega=\beta_{0} \alpha(s) /(4 \pi)=\left(11 N_{c}-2 N_{f}\right) \alpha(s) /(12 \pi)$. If we additionally substitute the scaling behavior of the renormalization constants $Z_{3}$ and $\tilde{Z}_{3}$, eqs. (24), and exploit the relation $\gamma+2 \delta+1=0$ we arrive at

$$
M(x)=m_{0}(L)+\frac{1}{\pi} \frac{\alpha(x)}{x} \int_{x_{0}}^{x} d y M(y)+\frac{1}{\pi} \int_{x}^{L} d y \frac{\alpha(y)}{y} M(y) .
$$

This well known equation describes the ultraviolet behavior of the quark mass function, see e.g. ref. [24]. Employing the perturbative form of the running coupling,

$$
\alpha(y)=\alpha(s)\left[\omega \log \left(\frac{y}{s}\right)+1\right]^{-1}
$$


one obtains in the chiral limit, $m_{0}(L)=0$, the so-called regular asymptotic form

$$
M(x)=\frac{2 \pi^{2} \gamma_{m}}{3} \frac{-\langle\bar{\Psi} \Psi\rangle}{x\left(\frac{1}{2} \ln \left(x / \Lambda_{Q C D}^{2}\right)\right)^{1-\gamma_{m}}} .
$$

Here $\langle\bar{\Psi} \Psi\rangle$ denotes the chiral condensate which is discussed in more detail in the next subsection. In the case of non-vanishing bare quark mass, $m_{0}(L) \neq 0$, the equation (31) is solved by the irregular asymptotic form,

$$
M(x)=M(s)\left[\omega \log \left(\frac{x}{s}\right)+1\right]^{-\gamma_{m}} .
$$

In this case we furthermore find

$$
\begin{aligned}
\gamma_{m} & =\frac{12}{11 N_{c}-2 N_{f}} \\
m_{0}(L) & =M(s)\left[\omega \log \left(\frac{L}{s}\right)+1\right]^{-\gamma_{m}}
\end{aligned}
$$

in accordance with perturbation theory.

We thus have shown that the bare vertex construction (14) admits a solution for the mass function $M(x)$ which has the correct perturbative behavior for large momenta. A similar analysis is possible for the DSE with the Curtis-Pennington type vertex, eq. (25). As the vector self energy goes to a constant in the limit of large momenta, eq. (23), all terms proportional to $A(x)-A(y)$ are suppressed in this limit. Furthermore, according to the perturbative expression (34) the $\Delta B$-term contributes at most subleading logarithmic corrections in eq. (25). The first term in the brackets reduces to the bare vertex form because $A(x, s) \approx A(y, s)$ for large momenta $x, y$. Thus we obtain the same ultraviolet limit from eq. (25) as for the bare vertex construction. This is certainly also the case if a Ball-Chiu type vertex is employed.

\section{THE QUARK PROPAGATOR IN QUENCHED QCD}

In this section we will compare quark propagator results in quenched approximation for three different vertex types, which share the non-Abelian part proposed in eq. (11) but differ in their Abelian parts. We will employ the bare vertex, eqs. (14), and the Curtis-Pennington (CP) type vertex, eqs. (13). Furthermore we use a Ball-Chiu (BC) type construction, which employs only the first three terms of the CP-vertex. In Landau gauge all these vertex ansätze satisfy the conditions (i) and (ii) formulated above eq. (11). In order to compare the different vertex types on a quantitative level we will calculate the pion decay constant $f_{\pi}$ and the chiral condensate from the respective solutions for the quark mass function.

Before doing so we have to specify the effective quark interaction as input from the YangMills sector.

\section{Effective quark interaction}

Via the ansatz of the quark-gluon vertex the effective quark interaction depends on the quark propagator functions $A(x, s)$ and $M(x)$ themselves. These functions will be determined 

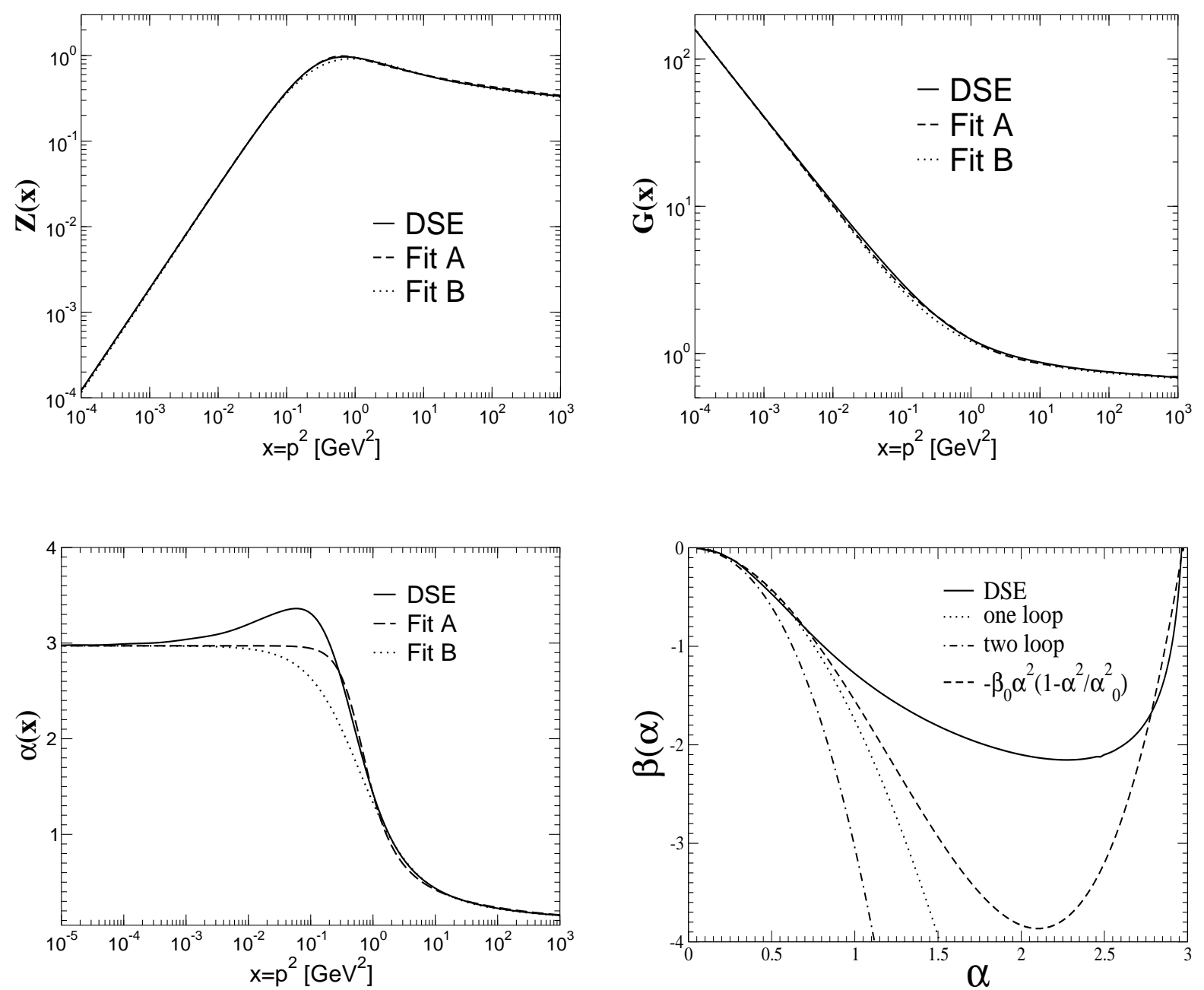

FIG. 3: Shown are the results for the gluon dressing function $Z$, the ghost dressing function $G$ and the running coupling $\alpha$. The two sets of fit functions are given in eqs. (37) and (38). The $\beta$-function corresponding to our DSE-solution is compared to the one- and two-loop expressions as well as to a polynomial in $\alpha$.

self-consistently in the process of the solution the quark DSE. As further input the running coupling and, if $d \neq 0$, the gluon and the ghost propagator are needed. These will be taken from the results of ref. [4].

The DSEs of the Yang-Mills sector are scale-independent and the underlying scale will be generated through dimensional transmutation during renormalization. To translate this scale into physical units the scale $\Lambda_{Q C D}$ is, in quenched approximation, determined from the large-momentum behavior of the running coupling, or more precisely, by requiring $\alpha\left(M_{Z}^{2}\right)=$ $\alpha\left((91.187 \mathrm{GeV})^{2}\right)=0.118$. Technically this is achieved by fitting the functional form of the 
running coupling. We employ two different fit functions [25] for the running coupling $\alpha(x)$ :

Fit A: $\quad \alpha(x)=\frac{\alpha(0)}{\ln \left[e+a_{1}\left(x / \Lambda_{Q C D}^{2}\right)^{a_{2}}+b_{1}\left(x / \Lambda_{Q C D}^{2}\right)^{b_{2}}\right]}$,

Fit B: $\quad \alpha(x)=\frac{1}{a+\left(x / \Lambda_{Q C D}^{2}\right)^{b}}[a \alpha(0)+$

$$
\left.\frac{4 \pi}{\beta_{0}}\left(\frac{1}{\ln \left(x / \Lambda_{Q C D}^{2}\right)}-\frac{1}{x / \Lambda_{Q C D}^{2}-1}\right)\left(x / \Lambda_{Q C D}^{2}\right)^{b}\right] .
$$

The value $\alpha(0)=8.915 / N_{c}$ is known from the infrared analysis. In both fits the ultraviolet behavior of the solution fixes the scale, $\Lambda_{Q C D}=0.71 \mathrm{GeV}$. Especially the fit B is very robust leading to a change in $\Lambda_{Q C D}$ of less than one percent when different fitting regions are chosen. Note that we have employed a MOM scheme, and thus $\Lambda_{Q C D}$ has to be interpreted as $\Lambda_{M O M}^{N_{f}=0}$, i.e. this scale has the expected magnitude [52]. Fit A employs the four additional parameters: $a_{1}=1.106, a_{2}=2.324, b_{1}=0.004, b_{2}=3.169$. Fit B has only two free parameters: $a=1.020, b=1.052$.

In Landau gauge the gluon and ghost propagators are defined by

$$
\begin{aligned}
D_{\mu \nu}(p) & =\left(\delta_{\mu \nu}-\frac{p_{\mu} p_{\nu}}{p^{2}}\right) \frac{Z\left(p^{2}\right)}{p^{2}} \\
D_{G}(p) & =-\frac{G\left(p^{2}\right)}{p^{2}}
\end{aligned}
$$

The gluon and ghost dressing functions, $Z\left(p^{2}\right)$ and $G\left(p^{2}\right)$, respectively, are then described by

$$
\begin{aligned}
& R(x)=\frac{c\left(x / \Lambda_{Q C D}^{2}\right)^{\kappa}+d\left(x / \Lambda_{Q C D}^{2}\right)^{2 \kappa}}{1+c\left(x / \Lambda_{Q C D}^{2}\right)^{\kappa}+d\left(x / \Lambda_{Q C D}^{2}\right)^{2 \kappa}}, \\
& Z(x)=\left(\frac{\alpha(x)}{\alpha(\mu)}\right)^{1+2 \delta} R^{2}(x), \\
& G(x)=\left(\frac{\alpha(x)}{\alpha(\mu)}\right)^{-\delta} R^{-1}(x),
\end{aligned}
$$

where $x=p^{2}$ and $c, d$ are fitting parameters for the auxiliary function $R(x)$. They are given by $c=1.269$ and $d=2.105$. Recall that the anomalous dimension $\gamma$ of the gluon is related to the anomalous dimension $\delta$ of the ghost by $\gamma=-1-2 \delta$ and $\delta=-9 / 44$ for the number of flavors $N_{f}=0$.

Whereas Fit $\mathrm{A}$ is better in the region $0.3 \mathrm{GeV}^{2}<x<1 \mathrm{GeV}^{2}$ where $\alpha$ is strongly rising, Fit $\mathrm{B}$ is slightly better in the region $1 \mathrm{GeV}^{2}<x<10 \mathrm{GeV}^{2}$. As can be seen in Fig. [3 both fits works very well and will be used as input below.

\section{Pion decay constant, chiral condensate and quark masses}

A correct calculation of the pion decay constants involves the pion Bethe-Salpeter amplitudes including the subleading components, see e.g. refs. 27, 28]. Apart from the dressed 
quark propagator the Bethe-Salpeter equation involves couplings between quarks and gluons. On the level of the quark DSE we have substituted the full quark-gluon vertex by an vertex ansatz. However, at present it is only known for certain cases how such a vertex ansatz in the quark DSE translates to the corresponding quark-gluon coupling in the Bethe-Salpeter equation [29]. No method is known up to know to derive the corresponding Bethe-Salpeter equation for dressed quark-gluon vertices as the BC- or CP-vertex constructions.

We thus have to rely on the approximation [30]

$$
f_{\pi}^{2}=-\frac{N_{c}}{4 \pi^{2}} Z_{2}(s, L) \int d y y \frac{M(y) A^{-1}(y, s)}{\left(y+M^{2}(y)\right)^{2}}\left(M(y)-\frac{y}{2} \frac{d M(y)}{d y}\right),
$$

which incorporates only the effects of the leading pion Bethe-Salpeter amplitude in the chiral limit 31]. From a comparison of the relative size of the amplitudes in model calculations [32, 33] one concludes that the approximation (42) should lead to an underestimation of $f_{\pi}$ by $10-20 \%$.

The renormalization point independent chiral condensate, $\langle\bar{\Psi} \Psi\rangle$, can be extracted from the ultraviolet behavior of the quark mass function in the chiral limit (c.f. eq. (33)):

$$
M(x) \stackrel{x \rightarrow L}{\longrightarrow} \frac{2 \pi^{2} \gamma_{m}}{3} \frac{-\langle\bar{\Psi} \Psi\rangle}{x\left(\frac{1}{2} \ln \left(x / \Lambda_{Q C D}^{2}\right)\right)^{1-\gamma_{m}}} .
$$

Hereby $\Lambda_{Q C D}$ is to be taken from a fit to the running coupling, c.f. eqs. (37), (38).

The renormalization point dependent chiral condensate $\langle\bar{\Psi} \Psi\rangle_{\mu}$ can be calculated via [28]

$$
-\langle\bar{\Psi} \Psi\rangle_{\mu}:=Z_{2}(s, L) Z_{m}(s, L) N_{c} \operatorname{tr}_{D} \int \frac{d^{4} q}{(2 \pi)^{4}} S_{c h}\left(q^{2}, s\right),
$$

where the trace is over Dirac indices, $S_{c h}$ is the quark propagator in the chiral limit and the squared renormalization point is denoted by $s=\mu^{2}$. To one-loop order both expressions for the condensate are connected by

$$
\langle\bar{\Psi} \Psi\rangle_{\mu}=\left(\frac{1}{2} \ln \left(\mu^{2} / \Lambda_{Q C D}^{2}\right)\right)^{\gamma_{m}}\langle\bar{\Psi} \Psi\rangle
$$

with $\gamma_{m}$ being the anomalous dimension of the quark mass function.

For the calculation of the chiral condensate we first have to determine the mass renormalization constant $Z_{m}(s, L)$. Recall the formal structure of the mass equation (15), which is given as

$$
M(x) A(x, s)=Z_{2}(s, L) Z_{m}(s, L) m_{R}(s)+Z_{2}(s, L) \Pi_{M}(x, s),
$$

where $\Pi_{M}(x, s)$ represents the dressing loop. In order to extract $Z_{m}(s, L)$ from this equation we have to clarify the meaning of $m_{R}(s)$ which is related to the unrenormalized mass by

$$
m_{0}(L)=Z_{m}(s, L) m_{R}(s),
$$

c.f. eq. (51). Evaluating eq. (46) at the perturbative momentum $x=s$ the matter seems clear. We achieve consistency with eqs. (34) and (36), if

$$
\begin{aligned}
m_{R}(s) & =M(s) \\
Z_{m}(s, L) & =\left[\omega \log \left(\frac{L}{s}\right)+1\right]^{-\gamma_{m}},
\end{aligned}
$$


which is indeed the correct perturbative scaling of the renormalization constant $Z_{m}$ [2].

Certainly one could implicitly define the finite parts of $Z_{m}$ such that the relation (48) holds in general for all renormalization points $s$. Then the parameter $m_{R}$ in the renormalized QCDLagrangian would already know about dynamical symmetry breaking. However, as the mass parameters of QCD are supposed to be generated in the electroweak sector of the standard model one could equally well argue that it is more systematic to exclude the effect of mass generation by strong interaction from $m_{R}$.

In our numerical calculations we will choose $s$ to be sufficiently large, therefore eq. (48) is valid anyway. Then $Z_{m}$ is determined by

$$
\begin{aligned}
Z_{m}(s, L) & =\frac{M(x) A(x, s)-Z_{2}(s, L) \Pi_{M}(x, s)}{Z_{2}(s, L) M(s)} \\
& =\frac{1}{Z_{2}(s, L)}-\frac{\Pi_{M}(s, s)}{M(s)} .
\end{aligned}
$$

For the last equation we have set $x=s$ and have used the renormalization condition $A(s, s)=$ 1.

In the numerical calculations we have to specify the masses $m_{R}(s)$ as input. Choosing a perturbative renormalization point $s$ allows one to evolve the masses $m_{R}(s)$ to a different scale $t$ by

$$
m_{R}(t)=m_{R}(s)\left(\frac{\ln \left(s / \Lambda_{Q C D}^{2}\right)}{\ln \left(t / \Lambda_{Q C D}^{2}\right)}\right)^{\gamma_{m}} .
$$

For $t=(2 \mathrm{GeV})^{2}$ typical values for the masses of the light quarks are given by the Particle Data Group [34]:

$$
\frac{1}{2}\left(m_{u}+m_{d}\right)(2 \mathrm{GeV}) \approx 4.5 \mathrm{MeV}, \quad m_{s}(2 \mathrm{GeV}) \approx 100 \mathrm{MeV}
$$

We will use similar masses in our calculations.

\section{Renormalization scheme and numerical method}

In the quark equation we employ a MOM regularization scheme similar to the one used for the ghost and gluon equations in refs. [4, 5]. For a fermion DSE this technique has already been used in quenched $\mathrm{QED}_{4}$ [35]. The formal structure of the quark equation is given by

$$
\begin{aligned}
A(x, s) & =Z_{2}(s, L)+Z_{2}(s, L) \Pi_{A}(x, s), \\
M(x) A(x, s) & =Z_{2}(s, L) Z_{m}(s, L) m_{R}(s)+Z_{2}(s, L) \Pi_{M}(x, s) .
\end{aligned}
$$

We eliminate $Z_{2}$ from the first equation by isolating it on the left hand side and subtracting the same equation for $x=s$. With

$$
\frac{1}{Z_{2}(s, L)}=\frac{1}{A(x, s)}+\frac{1}{A(x, s)} \Pi_{A}(x, s),
$$

we then have

$$
\frac{1}{A(x, s)}=1-\frac{1}{A(x, s)} \Pi_{A}(x, s)+\Pi_{A}(s, s),
$$


using the renormalization condition $A(s, s)=1$. In each iteration step we determine $A(x)$ from eq. (56) and subsequently $Z_{2}$ from eq. (55). As a numerical check we determine $Z_{2}$ at different momenta $x=p^{2}$. In our calculations we find $Z_{2}$ to be independent of $p^{2}$ to a high accuracy. For the mass function $M(x)$ we use

$$
M(x) A(x, s)=Z_{2}(s, L) \Pi_{M}(x, s)
$$

in the chiral limit and the subtracted equation

$$
M(x) A(x, s)=M(s)+Z_{2}(s, L) \Pi_{M}(x, s)-Z_{2}(s, L) \Pi_{M}(s, s)
$$

if chiral symmetry is broken explicitly, i.e. $m_{0} \neq 0$.

For the numerical iteration we employ a Newton method and represent the dressing functions $A(x)$ and $M(x)$ with the help of Chebychev polynomials. Furthermore, we use a numerical infrared cutoff $\epsilon$, which is taken small enough for the numerical results to be independent of $\epsilon$. Numerical difficulties arise in the case of the Curtis-Pennington type vertex and even more for the Ball-Chiu construction. If the external momentum $x$ and the loop momentum $y$ are both small and close to each other then the derivative-like terms

$$
\Delta A=\frac{A(x, s)-A(y, s)}{x-y}, \quad \Delta B=\frac{B(x, s)-B(y, s)}{x-y},
$$

are hard to evaluate accurately. Although the functions $A(x)$ and $B(x)$ are constant in the infrared and consequently should have derivatives close to zero one encounters large values for $\Delta A$ and $\Delta B$ due to numerical inaccuracies in $A$ and $B$. In order to evaluate $\Delta A$ and $\Delta B$ much more precisely at small momenta we fit the expressions

$$
A(x, s)=\frac{A(0, s)}{1+a_{1}\left(x / \Lambda_{Q C D}^{2}\right)^{a_{2}}}, \quad B(x, s)=\frac{B(0, s)}{1+b_{1}\left(x / \Lambda_{Q C D}^{2}\right)^{b_{2}}},
$$

with the parameters $a_{1}, a_{2}, b_{1}$ and $b_{2}$ to the numerically evaluated functions. The scale $\Lambda_{Q C D}=0.71 \mathrm{GeV}$ has been determined from the fits to the running coupling already in the last subsection.

For $x-y$ smaller than a suitable matching point we calculate the terms $\Delta A$ and $\Delta B$ from the fits. This procedure eliminates the numerical errors in the derivative terms and smoothes the numerical results considerably. In the case of the Ball-Chiu type vertex the iteration process does not converge unless we use these fits.

The renormalization condition employed in the ghost-gluon system of equations is $G^{2}(s) Z(s)=1$ with $\alpha(s)=0.118$ at the squared renormalization point $s=\mu^{2}$. Furthermore we choose a transversal tensor to contract the gluon equation, c.f. ref. [4]. The physical scale in the quenched calculations is taken directly from the Yang-Mills results, i.e. we use the experimental value $\alpha\left(M_{Z}^{2}\right)=0.118$ of the running coupling at the mass of the Z-boson to fix the scale.

\section{Numerical results}

In Fig. [ we give our numerical solutions for the quark mass function and the inverse vector self energy in the chiral limit, employing 'Fit A' for the effective quark interaction. We compare results obtained with five different ansätze for the quark-gluon vertex. For the 

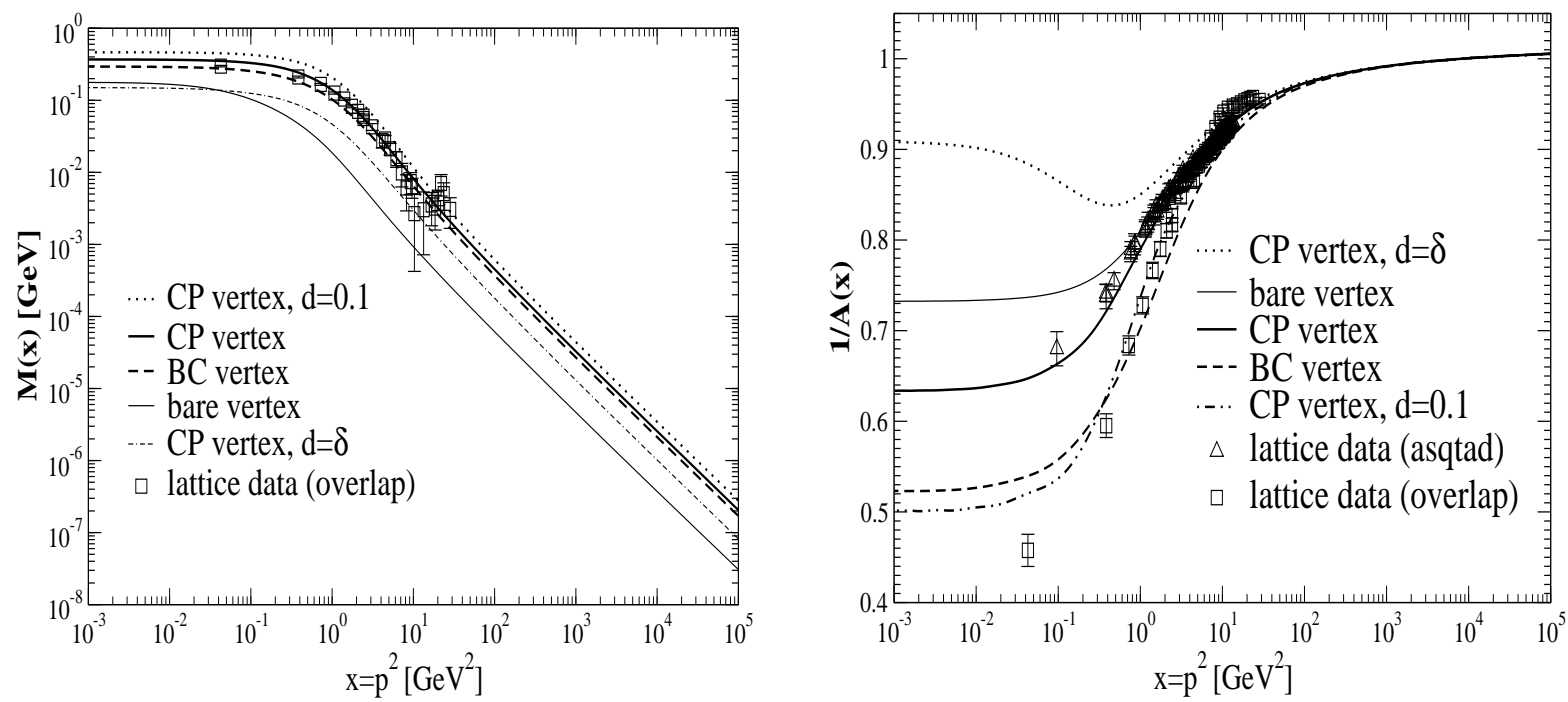

FIG. 4: The mass function $M(x)$ and the inverse vector self energy $1 / A(x)$ of a chiral quark are shown. We compare the results for five different vertices with lattice data taken from refs. 13, 14].

\begin{tabular}{c|c|c|c|c|c|c|c|c|c} 
& $\begin{array}{c}\mathrm{M}(0) \\
{[\mathrm{MeV}]}\end{array}$ & $\begin{array}{c}f_{\pi} \\
{[\mathrm{MeV}]}\end{array}$ & $\begin{array}{c}(-\langle\bar{\Psi} \Psi\rangle)^{1 / 3} \\
{[\mathrm{MeV}](\text { calc. })}\end{array}$ & $\begin{array}{c}(-\langle\bar{\Psi} \Psi\rangle)^{1 / 3} \\
{[\mathrm{MeV}] \text { (fit) }}\end{array}$ & $A^{-1}\left(0, M_{Z}^{2}\right)$ & $a_{1}$ & $a_{2}$ & $b_{1}$ & $b_{2}$ \\
\hline bare vertex & 177 & 38.5 & 162 & 160 & 0.733 & 3.05 & 0.99 & 0.06 & 1.00 \\
CP d= $\delta$ & 150 & 50.5 & 223 & 225 & 0.910 & - & - & - & - \\
BC-vertex & 293 & 62.6 & 276 & 284 & 0.523 & 1.10 & 0.99 & 0.29 & 0.92 \\
CP-vertex & 369 & 78.7 & 303 & 300 & 0.634 & 0.83 & 0.99 & 0.20 & 1.00 \\
CP d=0.1 & 464 & 87.5 & 334 & 330 & 0.501 & 0.79 & 0.99 & 0.34 & 0.95
\end{tabular}

TABLE I: The mass $M(0)$, the pion decay constant $f_{\pi}$ calculated with eq. (42), the renormalization point independent chiral condensate calculated with eqs. (44) and (45), and the condensate obtained by fitting the expression (43) to the chiral mass function in the ultraviolet for all five vertex types. Recall $\delta=-9 / 44$ in quenched approximation. If not stated otherwise the parameter $d$ in the vertex construction is taken to be $d=0$. For the case of the CP-vertex with $d=\delta$ we did not get good fits in the infrared.

generalized CP-vertex we investigate the 'natural' case $d=0$, the value $d=\delta=-9 / 44$, already adopted in refs. [20, 21], and the value $d=0.1$. Furthermore we employed the bare vertex construction and a Ball-Chiu type vertex. The corresponding masses at the momentum $p^{2}=0$, the pion decay constant $f_{\pi}$, the renormalization point independent chiral condensate and the fit parameters for the functions (60) are displayed in table I.

The numerical results for the mass function all have a characteristic plateau in the infrared and show the regular asymptotic behavior for large momenta, c.f. eq. (43). The bare vertex construction and the CP type vertex with $d=\delta$ both generate masses much smaller than typical phenomenological values of $300-400 \mathrm{MeV}$. The BC- and the CP-type construction with $d=0$ provide good results, whereas the choice $d=0.1$ leads to a somewhat large mass. The lattice calculations taken from refs. [13] (overlap fermions) and [14] (improved staggered action, Asqtad) favor masses around $300 \mathrm{MeV}$ with the caveat that they are obtained by an extrapolation from sizeable bare quark masses to the chiral limit. The numerical solutions for 

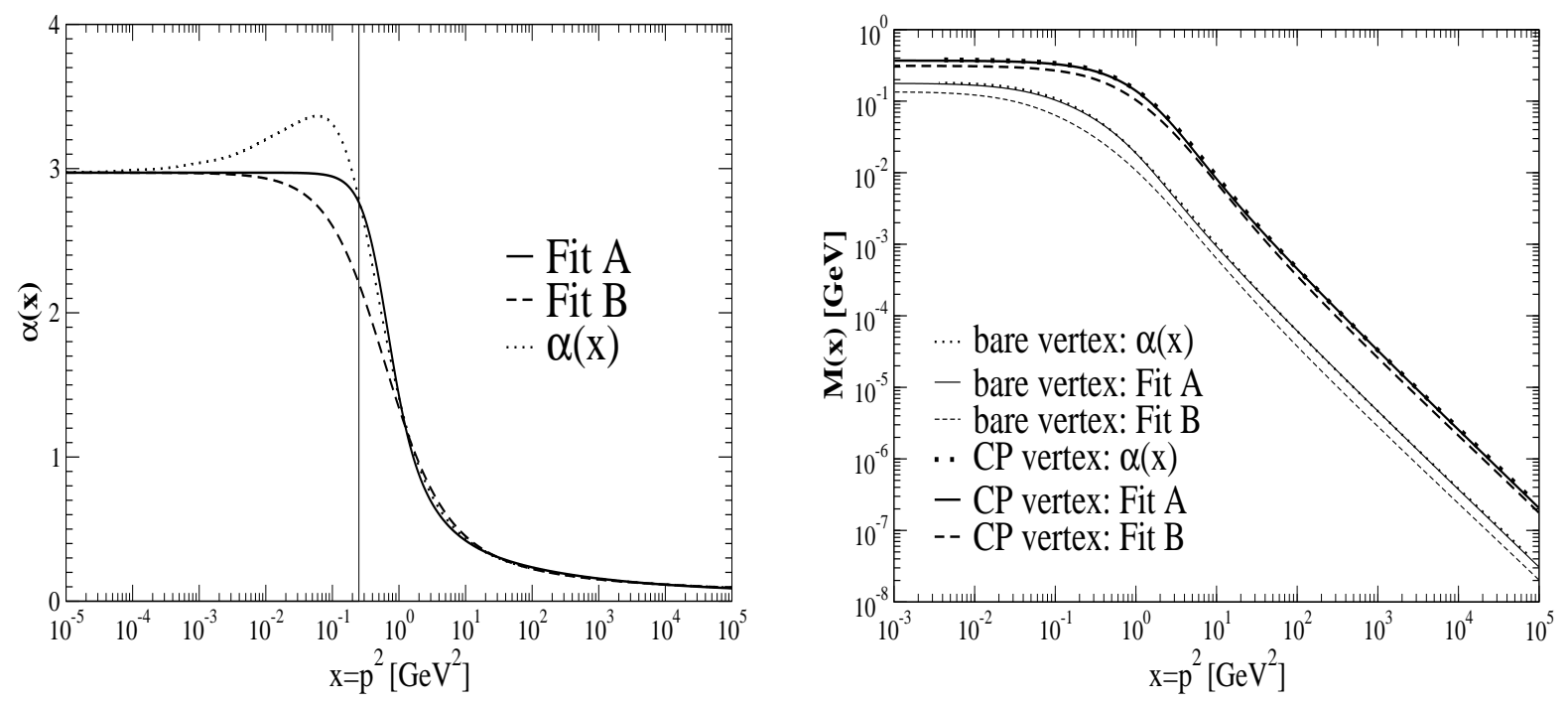

FIG. 5: Results for three different forms of the running coupling in the quark equation: The running coupling calculated in ref. [4] and the two fits given in eqs. (37), (38).

the wave function renormalization $1 / A$ can be seen in the right diagram of Fig. 4. Whereas the ultraviolet asymptotic behavior of all vertex constructions is similar we observe sizeable differences for small momenta. Again the bare vertex construction and the CP-vertex with $d=\delta$ are clearly disfavored by the lattice data.

Our approximate calculation of the pion decay constant should underestimate the experimental value $f_{\pi}=93 \mathrm{MeV}$ by $10-20 \%$, c.f. the discussion below eq. (42). We thus have best results for the CP-vertex construction with $d=0$ and $d=0.1$. Furthermore we obtain very good agreement between the two different methods to extract the chiral condensate. Compared to the phenomenological value $(-\langle\bar{\Psi} \Psi\rangle)^{1 / 3} \approx 250 \mathrm{MeV}$ most of our results are larger.

Apart from the case $d=\delta$ we obtain very good fits for the scalar and vector self energy, $A(x)$ and $B(x)$, for small momenta. The results for the parameters $a_{1}, a_{2}, b_{1}, b_{2}$ in the fit functions given by eq. (60) can be found in table I. It is interesting to note that the exponents $a_{2}$ and $b_{2}$ are found to be very close to one. Such a behavior could indicate a simple underlying functional form of the quark propagator. This will be explored in future work by a numerical continuation of our results to negative $p^{2}$, i.e. timelike momenta.

Fig. 5 compares results for the bare vertex and the CP-type construction with $d=0$ for three different forms of the running coupling in the interaction kernel of the quark equation. The two fit-functions, 'Fit A' and 'Fit B', have been given in eqs. (37), (38). Furthermore we used the running coupling calculated from the quenched ghost and gluon DSEs in ref. [4]. Although there is the (presumably) artificial bump at $p^{2} \approx 0.1 \mathrm{GeV}^{2}$ in the running coupling, the mass functions obtained from the DSE-result and from 'Fit A' are virtually indistinguishable. 'Fit B', however leads to somewhat smaller masses. This observation suggests that nearly all the dynamically generated mass is produced from the integration strength above $p=500 \mathrm{MeV}$, indicated by the vertical line in the plot of the running coupling. This is a favorable result as it would have been very unsatisfying if the artificial bump contributed a considerable amount to the quark mass function. 

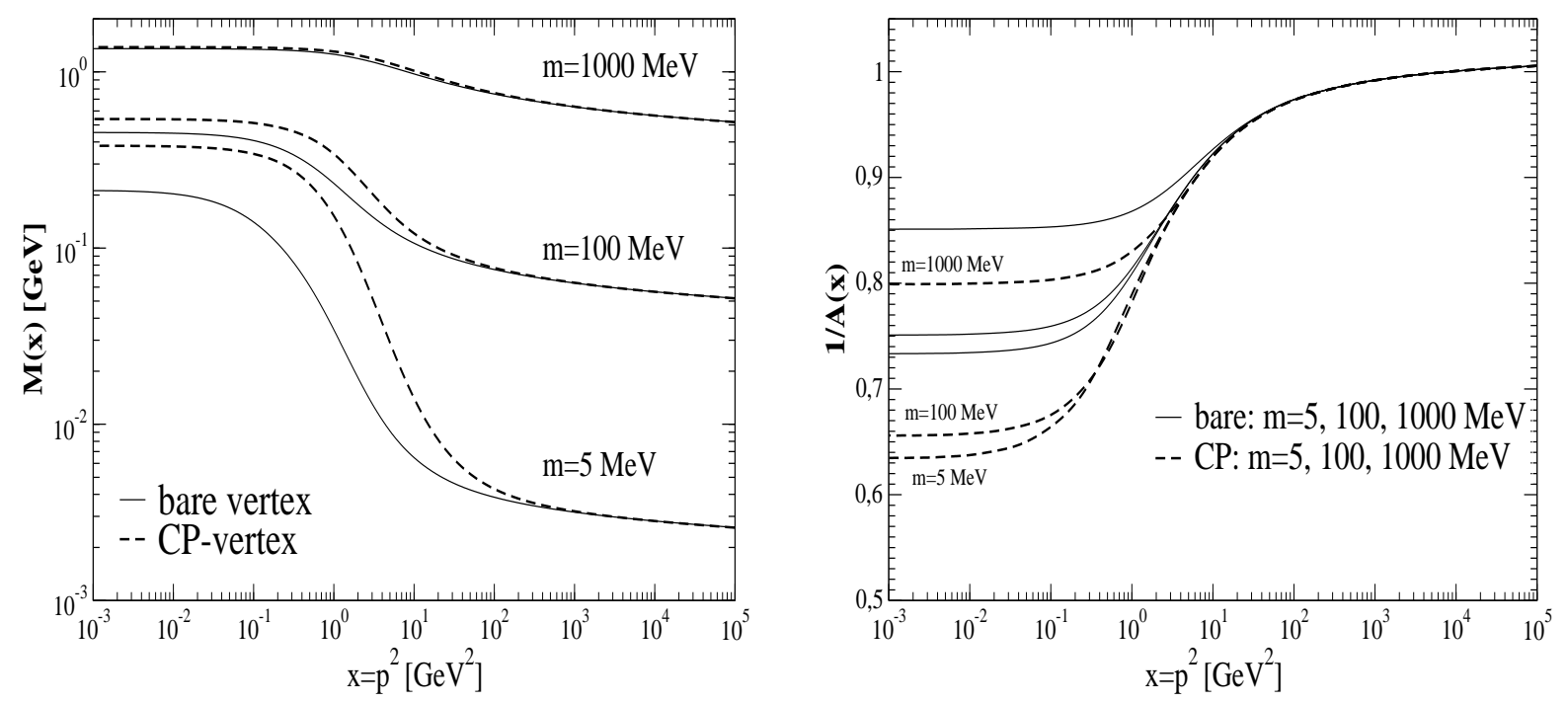

FIG. 6: These diagrams show our results when three different bare quark masses are employed. In the diagram on the right small quark masses correspond to small values for $1 / A$ in the infrared.

Finally we observe the effects of explicit chiral symmetry breaking in the plots of Fig. [6. We give results for three different quark masses, $m(2 \mathrm{GeV})=5 \mathrm{MeV}, m(2 \mathrm{GeV})=100$ $\mathrm{MeV}$ and $m(1 \mathrm{GeV})=1000 \mathrm{MeV}$. These values correspond roughly to the ones given by the Particle Data Group for the up/down-quark, the strange-quark and the charm-quark 34]. For small momenta we note again that the dressed vertex generates more mass in the quark equation than the bare vertex construction. This effect becomes much less dominant for the heavy quarks, where more and more of the infrared mass stems from explicit chiral symmetry breaking and not from dynamical mass generation. Furthermore in accordance with the analytical determination we observe the same ultraviolet behavior of the mass function for both vertex constructions.

\section{THE QUARK-LOOP IN THE GLUON DSE}

In this section we focus on the inclusion of the back-reaction of the quarks on the ghostgluon system, i.e. we will finally solve the complete set of mutually coupled Dyson-Schwinger equations for the quark, gluon and ghost propagator. To this end we incorporate the quarkloop in the truncation scheme for the gluon DSE which has been developed in ref. [4].

The formal structure of the gluon equation is given by (c.f. fig. (1)

$$
[D(p)]_{\mu \nu}^{-1}=Z_{3}\left[D^{(0)}(p)\right]_{\mu \nu}^{-1}+\Pi_{\mu \nu}^{\text {ghost }}(p)+\Pi_{\mu \nu}^{\text {gluon }}(p)+\Pi_{\mu \nu}^{\text {quark }}(p) .
$$

The contributions from the ghost- and gluon-loop, $\Pi_{\mu \nu}^{\text {ghost }}(p)$ and $\prod_{\mu \nu}^{\text {gluon }}(p)$, are treated in detail in ref. [4]. The contribution of the quark-loop to the gluon equation is given by

$$
\Pi_{\mu \nu}^{q u a r k}(p)=-\frac{g^{2} N_{f}}{2(2 \pi)^{4}} Z_{1 F} \int d^{4} q \operatorname{Tr}\left\{\gamma_{\mu} S(q) \Gamma_{\nu}(q, p) S(k)\right\}
$$




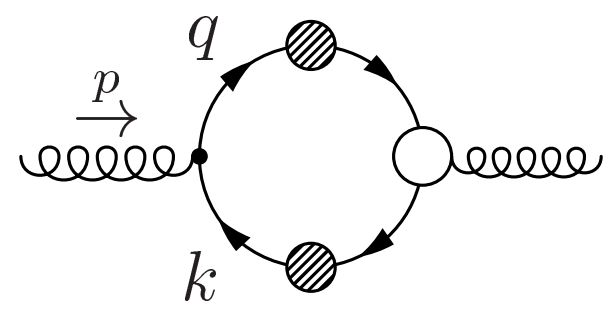

FIG. 7: Diagrammatical representation of the quark-loop in the Dyson-Schwinger equation for the gluon propagator.

where $p$ is the external gluon momentum and $q$ and $k=(q-p)$ are the momenta of the two quarks running in the loop, (cf. fig. 17). The trace is over Dirac indices.

In eqs. (11, 13) we have proposed an effective quark-gluon vertex $\Gamma_{\nu}$ with Abelian and non-Abelian parts such that the quark equation is multiplicatively renormalizable and oneloop perturbation theory is recovered for large momenta. However, this construction is not capable to account as well for the one-loop behavior of the unquenched gluon equation unless we switch the momentum arguments of the non-Abelian part $W^{\neg a b e l}$, eq. (11), from the gluon momentum to quark momenta. In the quark equation such a change of momentum arguments would either break Lorentz symmetry by preferring one quark line of the quarkgluon vertex or changes the ultraviolet behavior of the quark equation. We therefore have to use different momentum assignments for the quark-loop and the quark equation. Certainly, this is a deficiency which has to be resolved by a more elaborate vertex construction in future work. The aim of the present study, however, is to present an effective construction which captures essential properties of the theory.

Taking care of symmetries we propose the following ansatz for the non-Abelian part of the quark-gluon vertex in the quark-loop:

$$
W_{\text {quark-loop }}^{\text {auarl }}(y, z, x)=G(y) G(z) \tilde{Z}_{3}(L) \frac{\left(G(y) \tilde{Z}_{3}(L)\right)^{-d-d /(2 \delta)}}{\left(Z(y) Z_{3}(L)\right)^{d / 2}} \frac{\left(G(z) \tilde{Z}_{3}(L)\right)^{-d-d /(2 \delta)}}{\left(Z(z) Z_{3}(L)\right)^{d / 2}} .
$$

Here $x=p^{2}$ is the squared gluon momentum, $y=q^{2}$ and $z=k^{2}=(q-p)^{2}$ are the squared quark momenta, and $L=\Lambda^{2}$ is the squared cutoff. The Abelian part $V_{\nu}^{a b e l}$ of the vertex, given in eqs. (1314), is symmetric with respect to the quark momenta as well.

Plugging the Curtis-Pennington type vertex into the quark-loop and contracting the free Lorenz-indices with the tensor (c.f. the treatment of the gluon DSE in ref. [4])

$$
\mathcal{P}_{\mu \nu}^{(\zeta)}(p)=\delta_{\mu \nu}-\zeta \frac{p_{\mu} p_{\nu}}{p^{2}}
$$


we obtain

$$
\begin{aligned}
\Pi_{\text {quark }}= & -\frac{g^{2} N_{f}}{(2 \pi)^{4}} Z_{2} \int d^{4} q \frac{G(y)}{y+M^{2}(y)} \frac{G(z)}{z+M^{2}(z)} \frac{\left(G(y) G(z) \tilde{Z}_{3}^{2}(L)\right)^{-d-d /(2 \delta)}}{\left(Z(y) Z(z) Z_{3}^{2}(L)\right)^{d / 2}} \times \\
& \times A^{-2}(y) A^{-2}(z)\left\{\frac{A(y)+A(z)}{2}\left(W_{1}(x, y, z) A(y) A(z)+W_{2}(x, y, z) B(y) B(z)\right)\right. \\
& +\frac{A(y)-A(z)}{2(y-z)}\left(W_{3}(x, y, z) A(y) A(z)+W_{4}(x, y, z) B(y) B(z)\right) \\
& +\frac{B(y)-B(z)}{y-z}\left(W_{5}(x, y, z) A(y) B(z)+W_{6}(x, y, z) B(y) A(z)\right) \\
+ & \left.\frac{(A(y)-A(z))(y+z)}{2\left((y-z)^{2}+\left(M^{2}(y)+M^{2}(z)\right)^{2}\right)}\left(W_{7}(x, y, z) A(y) A(z)+W_{8}(x, y, z) B(y) B(z)\right)\right\},
\end{aligned}
$$

with the kernels

$$
\begin{aligned}
W_{1}(x, y, z)= & \frac{\zeta z^{2}}{3 x^{2}}+z\left(\frac{2-\zeta}{3 x}-\frac{2 \zeta y}{3 x^{2}}\right)-\frac{2}{3}+\frac{(2-\zeta) y}{3 x}+\frac{\zeta y^{2}}{3 x^{2}}, \\
W_{2}(x, y, z)= & \frac{2(4-\zeta)}{3 x}, \\
W_{3}(x, y, z)= & \frac{\zeta z^{3}}{3 x^{2}}-z^{2}\left(\frac{1+\zeta}{3 x}+\frac{\zeta y}{3 x^{2}}\right)+z\left(\frac{1}{3}+\frac{(2 \zeta-6) y}{3 x}-\frac{\zeta y^{2}}{3 x^{2}}\right) \\
& +\frac{y}{3}-\frac{(\zeta+1) y^{2}}{3 x}+\frac{\zeta y^{3}}{3 x^{2}}, \\
W_{4}(x, y, z)= & \frac{-2 \zeta z^{2}}{3 x^{2}}+z\left(\frac{4}{3 x}+\frac{4 \zeta y}{3 x^{2}}\right)-\frac{2}{3}+\frac{4 y}{3 x}-\frac{2 \zeta y^{2}}{3 x^{2}}, \\
W_{5}(x, y, z)= & \frac{\zeta z^{2}}{3 x^{2}}-z\left(\frac{1+\zeta}{3 x}+\frac{2 \zeta y}{3 x^{2}}\right)+\frac{1}{3}+\frac{(\zeta-3) y}{3 x}+\frac{\zeta y^{2}}{3 x^{2}}, \\
W_{6}(x, y, z)= & \frac{\zeta z^{2}}{3 x^{2}}-z\left(\frac{3-\zeta}{3 x}+\frac{2 \zeta y}{3 x^{2}}\right)+\frac{1}{3}+\frac{(-\zeta-1) y}{3 x}+\frac{\zeta y^{2}}{3 x^{2}}, \\
W_{7}(x, y, z)= & -\frac{z^{2}}{x}+z+\frac{y^{2}}{x}-y, \\
W_{8}(x, y, z)= & 2\left(-\frac{z}{x}+\frac{y}{x}\right) \cdot
\end{aligned}
$$

Note that the symmetry factor $1 / 2$ and a factor $1 /(3 x)$ from the left hand side of the gluon equation have been absorbed in the kernels. From this expression the corresponding one for the bare vertex construction can be read off easily by setting $W_{3-8}=0$ and replacing the remaining factor $(A(y)+A(z)) / 2$ in eq. (65) by unity.

\section{Ultraviolet analysis of the quark-loop}

It is long known that the introduction of a cutoff $\Lambda$ in the gluon DSE results in artificial quadratic divergencies due to the violation of gauge invariance. Certainly, to recover the 
correct perturbative limit of the gluon propagator such terms have to be removed from the gluon equation by a suitable regularization procedure. Quadratic divergencies only occur in the part of the inverse gluon propagator proportional to $\delta_{\mu \nu}$. Therefore one way to eliminate the quadratic divergencies is to project onto the part proportional to $p_{\mu} p_{\nu}$ [36] by choosing $\zeta=4$ in the projection tensor (64).

Another unambiguous way is to subtract the quadratically divergent terms from the kernel by hand. This procedure is valid for general $\zeta$ and allows to estimate the influence of spurious longitudinal terms in the right hand side of the gluon equation on the solutions by varying the parameter $\zeta$. Certainly in a perfect truncation scheme the solution should be independent of $\zeta$.

In ref. [4] it has been described in detail how to remove the quadratic divergencies in the ghost- and gluon-loop. Therefore in the following we concentrate on the quark-loop. To identify the divergent terms we expand the dressing functions in the integrand of the quark-loop around large loop momenta $y$ with the difference $(z-y)$ still larger than any quark mass.

To leading order this expansion amounts in the replacements

$$
\begin{aligned}
G(z) & \rightarrow G(y), \\
A(z) & \rightarrow A(y), \\
\frac{A(y)-A(z)}{y-z} & \rightarrow A^{\prime}(y), \\
\frac{B(y)-B(z)}{y-z} & \rightarrow B^{\prime}(y), \\
\frac{(A(y)-A(z))(y+z)}{2\left((y-z)^{2}+\left(M^{2}(y)+M^{2}(z)\right)^{2}\right)} & \rightarrow \frac{A^{\prime}(y)(y+z)}{2(y-z)},
\end{aligned}
$$

with the derivatives $A^{\prime}$ and $B^{\prime}$. Note that the first two equations are identical to the angular approximation employed previously in the ultraviolet analysis of the quark equation. For large momenta $x$ and $z$ the denominators in eq. (65) simplify and the angular integrals are trivially performed using the integrals given in appendix A. We arrive at

$$
\begin{aligned}
\Pi_{\text {quark }}^{U V}=-\frac{g^{2} N_{f}}{16 \pi^{2}} Z_{2} & \int d y G^{2}(y) \frac{G(y)^{-2 d-d / \delta}}{Z(y)^{d}} A^{-2}(y) \times \\
& \left\{A(y)\left(\frac{-2}{3 y}+\frac{4-\zeta}{3 x}+\frac{2(4-\zeta)}{3 x y} M^{2}(y)\right)\right. \\
& +\frac{A^{\prime}(y)}{2}\left(\frac{1}{3}+\frac{-2(4-\zeta) y}{3 x}+\left(\frac{-2}{3 y}+\frac{2(4-\zeta)}{3 x}\right) M^{2}(y)\right) \\
& \left.+B^{\prime}(y) M(y)\left(\frac{2}{3 y}-\frac{4-\zeta}{3 x}\right)+\frac{A^{\prime}(y)}{2}\left(\frac{4 y}{x}-1+\frac{4}{x} M^{2}(y)\right)\right\} .
\end{aligned}
$$

Keeping in mind a factor $(1 / y)$ hiding in the derivatives we identify three quadratically divergent terms: $(4-\zeta) / 3 x$ in the second line, $-2(4-\zeta) y / 3 x$ in the third line and $4 y / x$ in the last line. The first two of them are artefacts of the regularization and will be subtracted from the kernels. However, we encounter the additional $\zeta$-independent quadratic divergent term $4 y / x$ originating from the transverse part of the Curtis-Pennington vertex. Such a term 
is already known from corresponding studies in QED [37]. Although first suggestions have been made how the Curtis-Pennington vertex should be modified to avoid this problem 3], a convincing solution has not been found yet. In our study we therefore choose the pragmatic strategy of subtracting this term by hand together with the other quadratically divergent parts.

Moreover we subtract all further terms proportional to $(4-\zeta)$. Although these terms are not quadratically divergent they are artefacts of the regularization. We then obtain a $\zeta$-independent expression for the quark loop at large momenta. In ref. [4] similar $\zeta$ independent expressions for the ghost- and gluon-loop have been derived. We therefore arrive at a transversal right hand side of the gluon equation for large momenta as required in Landau gauge.

Collecting all modifications together we have the new kernels

$$
\begin{aligned}
& \widetilde{W}_{1}(x, y, z)=W_{1}(x, y, z)-\frac{(y+z)(4-\zeta)}{6 x}, \\
& \widetilde{W}_{2}(x, y, z)=0 \\
& \widetilde{W}_{3}(x, y, z)=W_{3}(x, y, z)+\frac{2 z y(4-\zeta)}{3 x}, \\
& \widetilde{W}_{4}(x, y, z)=W_{4}(x, y, z)-\frac{(y+z)(4-\zeta)}{3 x}, \\
& \widetilde{W}_{5}(x, y, z)=W_{5}(x, y, z)-\frac{(y+z)(4-\zeta)}{6 x}, \\
& \widetilde{W}_{6}(x, y, z)=W_{6}(x, y, z)-\frac{(y+z)(4-\zeta)}{6 x}, \\
& \widetilde{W}_{7}(x, y, z)=W_{7}(x, y, z)-\frac{(y-z)(y+z)}{x}, \\
& \widetilde{W}_{8}(x, y, z)=W_{8}(x, y, z) .
\end{aligned}
$$

Note that the subtracted terms are chosen to preserve the symmetry of the kernels with respect to the squared quark momenta $y$ and $z$.

Without quadratic divergences we are in a position to extract the leading logarithmic divergence of the quark-loop. With modified kernels the ultraviolet limit of the quark-loop is given by

$$
\begin{aligned}
\Pi_{\text {quark }}^{U V}=-\frac{g^{2} N_{f}}{16 \pi^{2}} Z_{2} \int & d y G^{2}(y) \frac{G(y)^{-2 d-d / \delta}}{Z(y)^{d}} A^{-2}(y) \times \\
& \left\{A(y) \frac{-2}{3 y}+\frac{A^{\prime}(y)}{2}\left(\frac{1}{3}+\frac{-2}{3 y} M^{2}(y)\right)\right. \\
+ & \left.B^{\prime}(y) M(y) \frac{2}{3 y}+\frac{A^{\prime}(y)}{2}\left(-1+\frac{4}{x} M^{2}(y)\right)\right\} .
\end{aligned}
$$

Similar to the situation in the DSE for the quark mass function the leading ultraviolet term is the first term in the curly brackets. For the ghost and gluon dressing functions we employ 
the perturbative ansatz

$$
\begin{aligned}
& G(z)=G(s)\left[\omega \log \left(\frac{z}{s}\right)+1\right]^{\delta}, \\
& Z(z)=Z(s)\left[\omega \log \left(\frac{z}{s}\right)+1\right]^{\gamma},
\end{aligned}
$$

and determine the anomalous dimensions $\delta$ and $\gamma$ as well as the coefficient $\omega$ selfconsistently as follows. Substituting the ultraviolet limit of the vector self energy, eq. (23)), and choosing the perturbative renormalization condition $G(s)=Z(s)=1$ we arrive at

$$
\Pi_{\text {quark }}^{U V}(p)=\frac{2 N_{f}}{3(2 \delta+1) \omega} \frac{g^{2}}{16 \pi^{2}}\left\{\left[\omega \log \left(\frac{L}{s}\right)+1\right]^{2 \delta+1}-\left[\omega \log \left(\frac{x}{s}\right)+1\right]^{2 \delta+1}\right\} .
$$

Combining this expression with the results for the ghost- and gluon-loop from ref. [4] we obtain as ultraviolet limit of the gluon equation

$$
\begin{gathered}
{\left[\omega \log \left(\frac{x}{s}\right)+1\right]^{-\gamma}=Z_{3}+\left(\frac{N_{c} g^{2}}{96 \pi^{2} \omega(2 \delta+1)}-\frac{7 N_{c} g^{2}}{48 \pi^{2} \omega(2 \delta+1)}+\frac{2 N_{f} g^{2}}{48 \pi^{2} \omega(2 \delta+1)}\right) \times} \\
\left\{\left[\omega \log \left(\frac{L}{s}\right)+1\right]^{2 \delta+1}-\left[\omega \log \left(\frac{x}{s}\right)+1\right]^{2 \delta+1}\right\} .
\end{gathered}
$$

The corresponding expression for the ghost equation reads [4]

$$
\begin{aligned}
{\left[\omega \log \left(\frac{x}{s}\right)+1\right]^{-\delta}=\tilde{Z}_{3} } & -\frac{3 N_{c} g^{2}}{64 \pi^{2} \omega(\gamma+\delta+1)} \times \\
& \left\{\left[\omega \log \left(\frac{L}{s}\right)+1\right]^{\gamma+\delta+1}-\left[\omega \log \left(\frac{x}{s}\right)+1\right]^{\gamma+\delta+1}\right\} .
\end{aligned}
$$

The renormalization constants $Z_{3}(s, L)$ and $\tilde{Z}_{3}(s, L)$ cancel the cutoff dependence, i.e. the respective first terms in the curly brackets. Thus, the power and the prefactor of the second term have to match with the left hand side of the equations. This leads to the anomalous dimensions

$$
\begin{aligned}
& \gamma=\frac{-13 N_{c}+4 N_{f}}{22 N_{c}-4 N_{f}} \\
& \delta=\frac{-9 N_{c}}{44 N_{c}-8 N_{f}}
\end{aligned}
$$

which are related by $\gamma+2 \delta+1=0$ and in accordance with one-loop perturbation theory for arbitrary numbers of colors $N_{c}$ and flavors $N_{f}$. For the coefficient $\omega$ one obtains

$$
\omega=\left(11 N_{c}-2 N_{f}\right) \alpha(s) /(12 \pi)=\beta_{0} \alpha(s) /(4 \pi) .
$$

When combined according to eq. (177) our ghost and gluon dressing functions lead to the correct one-loop running of the coupling $\alpha(x)$ at large momenta. 


\section{Infrared analysis of the quark-loop}

The infrared analysis of the ghost and gluon DSEs in a truncation with bare ghost-gluon vertex has been performed in refs. [4, 7, 8]. To leading order the power law ansatz

$$
Z(x) \sim x^{2 \kappa}, \quad G(x) \sim x^{-\kappa},
$$

for the ghost and gluon dressing functions at small momenta $x=p^{2}$ has been employed. For a transverse projection tensor (64), i.e. $\zeta=1$, one obtains $\kappa=(93-\sqrt{1201}) / 98 \approx 0.5954$ for the exponent of the dressing functions and subsequently the fixed point $\alpha(0) \approx 8.915 / N_{c}$ for the running coupling in the infrared.

These results have been obtained in a truncation where the ghost-loop dominates the gluon-loop in the infrared. Therefore in order to investigate the effects of dynamical quarks in the system we compare the infrared behavior of the quark-loop with the one of the ghostloop. Substituting the ansatz (91) into the gluon equation and calculating the ghost-loop along the lines of the infrared analysis given in refs. [4, 7] one finds the ghost-loop to be proportional to $x^{-2 \kappa}$. For the quark-loop including the effects of dynamically generated quark masses we obtain

$$
\Pi_{\text {quark }}^{I R}(p) \sim x^{-2 \kappa+2+\kappa d / \delta} .
$$

Therefore the quark-loop is suppressed for small momenta provided the parameter $d$ fulfills the condition

$$
d<\frac{-2 \delta}{\kappa} \text {. }
$$

As we have $\kappa \approx 0.5954$ and $\delta=-1 / 4$ for $N_{c}=3$ and $N_{f}=3$ we find the condition $d<0.84$, which is satisfied for all quark-gluon vertices employed in our calculation. From a numerical point of view we encounter serious instabilities in the quark equation once $d$ is taken to be larger than $d \approx 0.2$.

We conclude that with dynamically generated quark masses the quark-loop does not change the infrared behavior of the ghost and gluon dressing functions found in refs. 7, 8]. In pure Yang-Mills theory as well as in QCD we thus have an infrared finite or vanishing gluon propagator and a ghost propagator which is more divergent than a simple pole. The Kugo-Ojima confinement criterion [38, 39] and Zwanziger's horizon condition [8, 40] are both fulfilled not only in pure Yang-Mills theory (see e.g. refs. 25]) but also in QCD.

The reasoning above reveals a selfconsistent picture valid for a small number of flavours: As has been demonstrated in our quenched calculations the combined strength of the ghost and gluon propagator generates sizeable dynamical quark masses in the quark DSE. In the unquenched case these masses suppress the quark loop in the infrared such that the ghost and gluon propagators are hardly changed in the infrared and in turn nearly the same amount of mass is generated in the quark equation as in the quenched case. This scenario is verified by our numerical calculations presented in the next section. On the other hand for a sufficiently large number of light flavours we expect a different selfconsistent picture to apply: Chiral symmetry should be restored. With vanishing quark masses the quark loop will contribute to the gluon DSE at small momenta and the infrared behaviour of the Yang-Mills sector will be changed. Especially it is expected that the value of the fixed point of the running coupling is decreased dramatically. This in turn drives the quark equation to the chirally symmetric solution with $M\left(p^{2}\right) \equiv 0$. This second selfconsistent scenario as well as the situation under the presence of a small amount of explicit chiral symetry breaking are subject to future investigations. 


\begin{tabular}{|c|c|c|c|c|c|c|c|c|c|c|}
\hline & \multicolumn{2}{|c|}{$\begin{array}{l}\mathrm{M}(0) \\
{[\mathrm{MeV}]}\end{array}$} & \multicolumn{2}{|c|}{$\begin{array}{c}f_{\pi} \\
{[\mathrm{MeV}]}\end{array}$} & \multicolumn{2}{|c|}{$\begin{array}{c}(-\langle\bar{\Psi} \Psi\rangle)^{1 / 3} \\
{[\mathrm{MeV}]}\end{array}$} & \multicolumn{2}{|c|}{$\begin{array}{c}\alpha\left(M_{Z}\right) \\
-\end{array}$} & \multicolumn{2}{|c|}{$\begin{array}{c}\Lambda_{Q C D}^{M O M} \\
{[\mathrm{MeV}]}\end{array}$} \\
\hline vertex & qu. & unqu. & qu. & unqu. & qu. & unqu. & qu. & unqu. & qu. & unqu. \\
\hline bare $\mathrm{d}=0$ & 177 & 176 & 38.5 & 38.4 & 160 & 170 & 0.118 & 0.146 & 710 & 748 \\
\hline $\mathrm{CP} d=\delta$ & 150 & 133 & 50.5 & 46.3 & 225 & 230 & 0.118 & 0.146 & 710 & 746 \\
\hline $\mathrm{CP} \mathrm{d}=0$ & 369 & 360 & 78.7 & 78.7 & 300 & 310 & 0.118 & 0.143 & 710 & 672 \\
\hline $\mathrm{CP} \mathrm{d}=0.1$ & 464 & 437 & 87.5 & 85.5 & 330 & 330 & 0.118 & 0.142 & 710 & 640 \\
\hline
\end{tabular}

TABLE II: A comparison between the quenched (qu.) and unquenched (unqu.) results for the quark mass $\mathrm{M}(0)$, the pion decay constant $f_{\pi}$, the renormalization point independent chiral condensate, the running coupling at the mass of the Z-boson and $\Lambda_{Q C D}^{M O M}$ for different vertices and values of the parameter $d$. The unquenched calculations are done for $N_{f}=3$ chiral quarks. Furthermore we have $\delta=-9 N_{c} /\left(44 N_{c}-8 N_{f}\right)=-0.25$ in the present case.

\section{NUMERICAL RESULTS}

The numerical treatment of the integrals in the ghost and gluon equations has been described in detail in ref. [4]. The iteration process is done for the ghost-gluon system and the quark equations separately: we first iterate the $N_{f}$ mutually uncoupled quark systems until convergence is achieved, feed the output into the ghost and gluon system, iterate until the ghost-gluon system converges, feed the output back into the quark equations and so on, until complete convergence of all equations is achieved. We renormalized at the point $s=\mu^{2}$ given by $\alpha(s)=0.2$ and used a transverse tensor to contract the gluon equation, $\zeta=1$.

In contrast to the quenched calculation we fix the physical scale of the system not by the condition $\alpha\left(M_{Z}^{2}\right)=0.118$ but by adjusting the pion decay constant. This choice has an important advantage: Whereas the behavior of the running coupling for large momenta depends strongly on $N_{f}$ the pion decay constant turns out to be almost independent of the number of flavors. In our quenched calculation we obtained $f_{\pi}=78.7 \mathrm{MeV}$ for the CP-type vertex with $d=0$. Recalling that our approximation (42) should lead to an underestimation of $f_{\pi}$ by about $10-20 \%$ this value is in good accordance with experiment as $f_{\pi}^{\exp }=93$ $\mathrm{MeV}$. We therefore chose the scale in the unquenched calculations to lead to the same decay constant for the CP-type vertex with $d=0$.

In table I we compare results for the quenched and unquenched system of equations. The quark mass, the pion decay constant and the chiral condensate differ only slightly for each vertex construction respectively. The only sizeable difference occurs in the running coupling. As expected from perturbation theory the unquenched running for $N_{f}=3$ results in larger values of the running coupling at $p^{2}=\left(M_{Z}\right)^{2}$ compared to the quenched case $N_{f}=0$. We obtain $\alpha\left(M_{z}\right) \approx 0.140$, which is somewhat larger than usually quoted values from experiment. However, such large values are not yet excluded by experiment. A recent analysis of experimental data from $\tau$-decay suggests $\alpha\left(M_{z}\right) \approx 0.129$ [41]. If we increase the number of flavors in our calculation we encounter large numerical uncertainties and do not obtain convergence for $N_{f} \geq 5$. This might be a signal that in the range $3<N_{f} \leq 5$ the above discussed transition of the system to the chirally symmetric phase takes place.

All employed vertex constructions allow for nontrivial solutions of the quark equation corresponding to dynamical chiral symmetry breaking. However, similar to the quenched case and in accordance with the results of refs. [20, 21] the bare vertex construction and the CP-type vertex with $d=\delta$ generate much too small quark masses compared with typical 

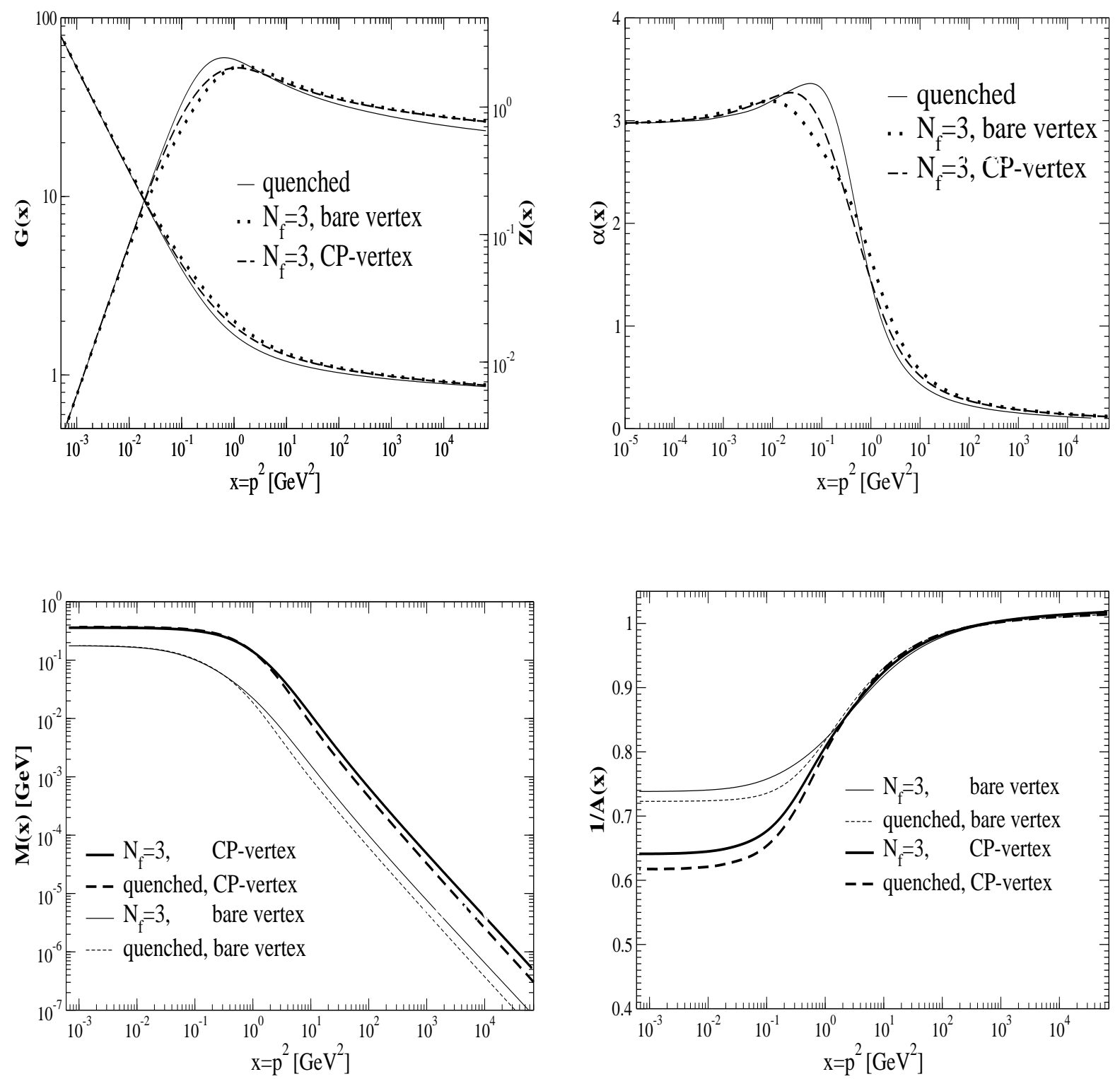

FIG. 8: Displayed are the ghost and gluon dressing function, $Z$ and $G$, the running coupling $\alpha$, the quark mass function $M$ and the inverse vector self energy $1 / A$. The calculations are done quenched and unquenched with $N_{f}=3$ quarks in the chiral limit. The parameter $d$ in the vertices is set to $d=0$.

phenomenological values. For $d=0$ we obtain good results for the quark mass, the pion decay constant and the chiral condensate, whereas the choice $d=0.1$ leads to somewhat large values. It is interesting to note that $d=0$ of all values is preferred as in this case the quark equation resembles most the fermion equation of QED.

In Fig. 8 we display the ghost, gluon and quark dressing functions corresponding to the unquenched and quenched cases with $d=0$ from table II 53]. We find different anomalous dimensions in the ultraviolet corresponding to the change from $N_{f}=0$ to $N_{f}=3$, c.f. eqs. (30), (33), (89). As expected from the infrared analysis of the quark-loop the backreaction of dynamical quarks in the gluon equation does not affect the infrared behavior of the ghost and gluon dressing functions. Consequently the infrared fixed point of the running 

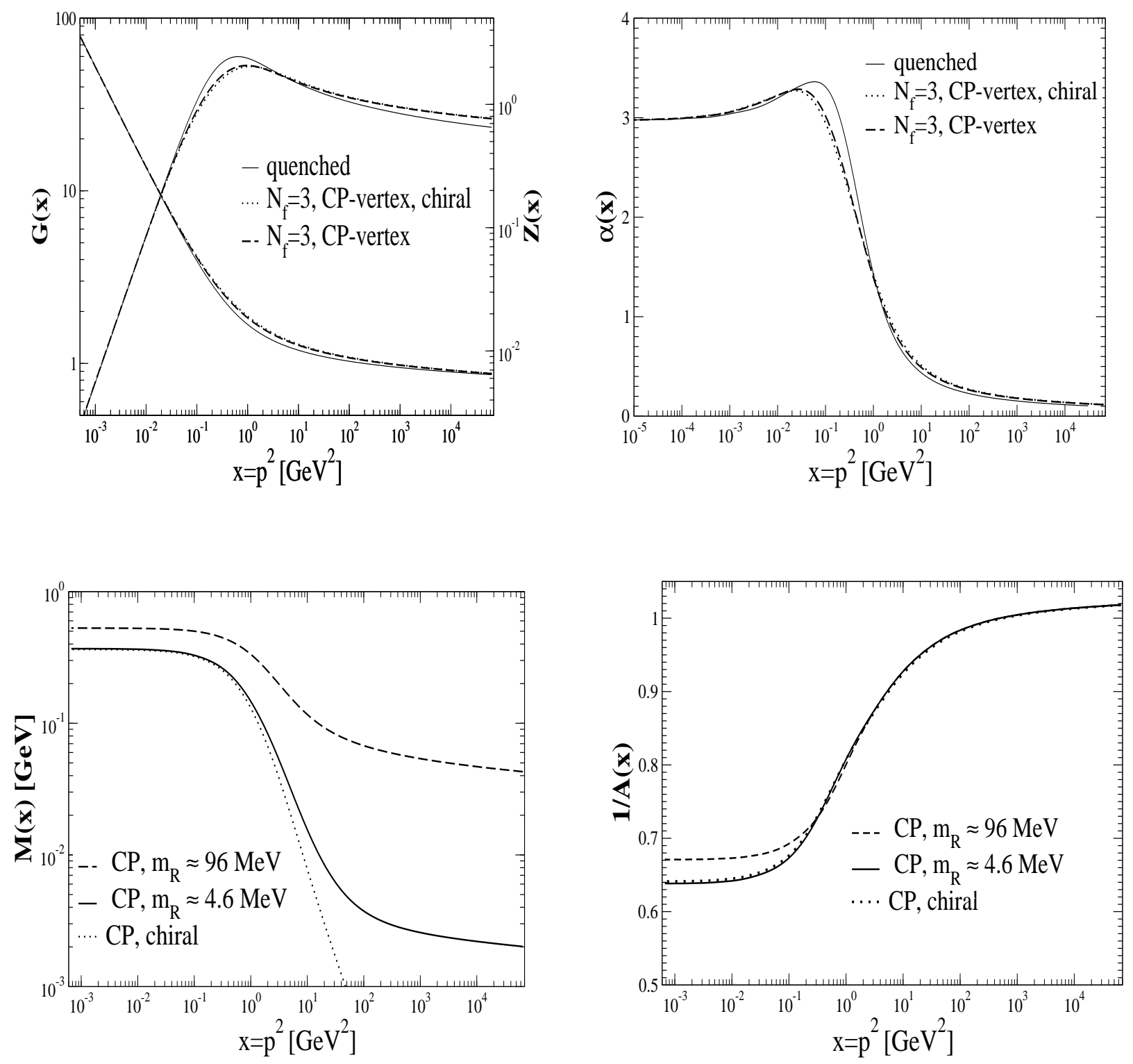

FIG. 9: Results from the unquenched calculation with $N_{f}=3$ massive quarks. We used the renormalized masses $m_{u / d}(2 \mathrm{GeV}) \approx 4.6 \mathrm{MeV}$ and $m_{s}(2 \mathrm{GeV}) \approx 96 \mathrm{MeV}$. Again we chose $d=0$ for both vertices.

coupling is the same as in pure Yang-Mills theory.

Our results for the case of explicitly broken chiral symmetry are shown in Fig. 9. We choose $N_{f}=3$ with renormalized quark masses corresponding to $m_{u / d}(2 \mathrm{GeV})=4.6 \mathrm{MeV}$ and $m_{s}(2 \mathrm{GeV})=96 \mathrm{MeV}$ within our renormalization scheme. These masses are well in the range suggested by the Particle Data Group [34], however, they should not be identified directly as the PDG employs an $\overline{M S}$-scheme. Compared to the chiral case the behavior of the ghost and gluon dressing functions hardly changes. For the quark mass function we obtain the irregular asymptotic solution in the ultraviolet as expected.

For further use, e.g. in phenomenological calculations, we provide fits to our results for 


\begin{tabular}{||c|c|c|c|c||c|c|c|c|c||}
$\begin{array}{c}\Lambda_{Q C D}^{M O M} \\
{[\mathrm{MeV}]}\end{array}$ & $\mathrm{a}$ & $\mathrm{b}$ & $\mathrm{c}$ & $\mathrm{d}$ & $\begin{array}{c}m_{R} \\
{[\mathrm{MeV}]}\end{array}$ & $\begin{array}{c}\hat{m} \\
{[\mathrm{MeV}]}\end{array}$ & $\begin{array}{c}\mathrm{M}(0) \\
{[\mathrm{MeV}]}\end{array}$ & $g_{1}$ & $g_{2}$ \\
\hline 625 & 1.22 & 1.00 & 1.33 & 2.01 & $\begin{array}{c}4.6 \\
96\end{array}$ & $\begin{array}{c}4.6 \\
98\end{array}$ & $\begin{array}{l}369 \\
528\end{array}$ & $\begin{array}{l}1.83 \\
2.96\end{array}$ & 1.23 \\
& & & & & 963
\end{tabular}

\begin{tabular}{||c|c|c|c|c||}
$A^{-1}(0, s)$ & $h_{1}$ & $h_{2}$ & $h_{3}$ & $h_{4}$ \\
& & & & \\
\hline 0.638 & 0.515 & 0.00688 & 0.562 & 0.00681 \\
0.671 & 0.302 & 0.00139 & 0.318 & 0.00137
\end{tabular}

TABLE III: Parameters for the fits to the unquenched results with $N_{f}=3, \delta=-0.25, \gamma_{m}=12 / 27$ and $\beta_{0}=27 / 3$, using the $\mathrm{CP}$-vertex with $d=0$. The renormalization point $s=497 \mathrm{GeV}^{2}$ is determined by the condition $\alpha(s)=0.2$. Note the change in $\Lambda_{Q C D}^{M O M}$ as compared to the case of chiral quarks.

the quark propagator employing the fit functions

$$
\begin{aligned}
& M(x)=\frac{1}{g_{1}+\left(x / \Lambda_{Q C D}^{2}\right)^{g_{2}}}\left(g_{1} M(0)+\right. \\
&\left.\hat{m}\left[\frac{2}{\ln \left(x / \Lambda_{Q C D}^{2}\right)}-\frac{2}{\left(x / \Lambda_{Q C D}^{2}\right)-1}\right]^{\gamma_{m}}\left(x / \Lambda_{Q C D}^{2}\right)^{g_{2}}\right), \\
& {[A(x, s)]^{-1}=\frac{[A(0, s)]^{-1}+h_{1}\left(x / \Lambda_{Q C D}^{2}\right)+h_{2}\left(x / \Lambda_{Q C D}^{2}\right)^{2}}{1+h_{3}\left(x / \Lambda_{Q C D}^{2}\right)+h_{4}\left(x / \Lambda_{Q C D}^{2}\right)^{2}}, }
\end{aligned}
$$

with $x=p^{2}$ and the six parameters $g_{1}, g_{2}, h_{1}, h_{2}, h_{3}, h_{4}$. We used the renormalization point independent current-quark mass $\hat{m}$, which is related to the renormalized mass $M(s)$ by

$$
\hat{m}=M(s)\left(\frac{1}{2} \ln \left[s / \Lambda_{Q C D}^{2}\right]\right)^{\gamma_{m}},
$$

to one loop order. For the running coupling, the ghost and the gluon dressing function we use the form 'Fit B', given in eq. (38) and the fit functions from eqs. (41). In table [II we give our values for all parameters as well as the numerical results for $M(0)$ and $[A(0)]^{-1}$. Note that the scale $\Lambda_{Q C D}^{M O M}$ is different to the corresponding scale in the chiral limit due to the different ultraviolet behavior of the quark-loop when quarks with non-vanishing bare masses are employed. When plotted the fits are virtually indistinguishable from our results in Fig. 9 .

Unquenched lattice calculations employing dynamical quarks are complex and time consuming [43]. To our knowledge such simulations for the propagators of QCD have not yet been performed. From our results in the Dyson-Schwinger approach we do not expect drastic differences between quenched and unquenched propagators on the lattice. Note, however, that our calculation includes quark-loop corrections to the gluon self energy but not higher order vertex corrections like mesonic loops. In a model calculation of the pion charge radius such loops have been estimated to contribute roughly at the order of ten percent [44].

Finally, we investigate possible positivity violations in the gluon and quark propagators. According to the Osterwalder-Schrader axiom of reflection positivity a two-point correlation 


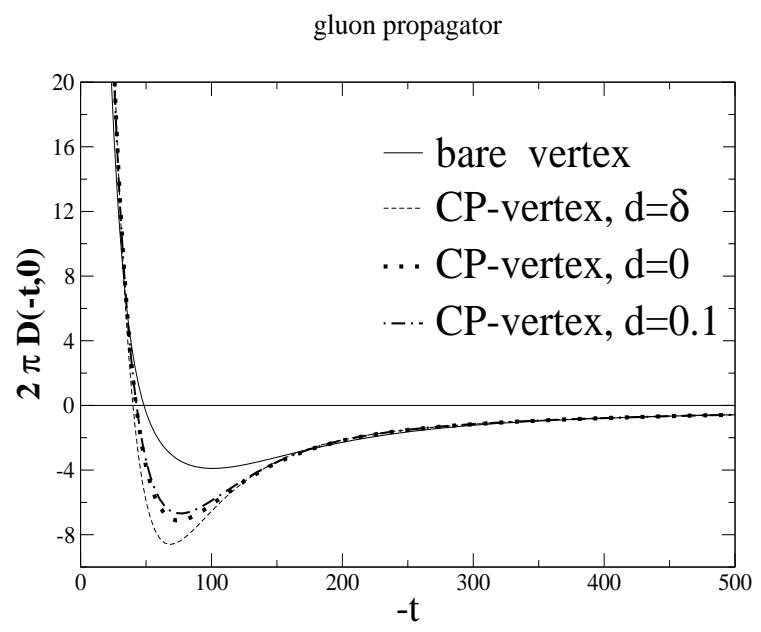

quark propagator: $\sigma_{\mathrm{S}}$

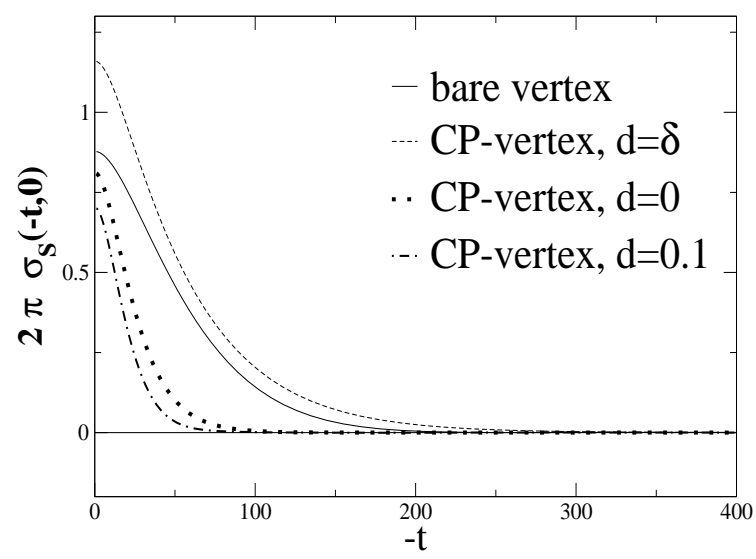

quark propagator: $\sigma_{\mathrm{V}}$

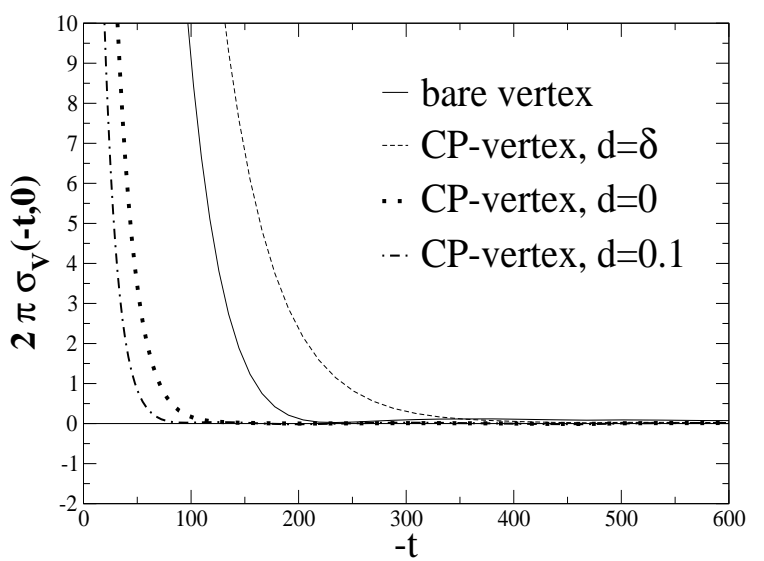

FIG. 10: Here we display the one dimensional Fourier transforms of the gluon propagator, $D\left(-t, \vec{p}^{2}\right)$, and the scalar and vector parts of the quark propagator, $\sigma_{S}\left(-t, \vec{p}^{2}\right)$ and $\sigma_{V}\left(-t, \vec{p}^{2}\right)$. For the three momentum we chose $\vec{p}^{2}=0$. The time $t$ is given in internal units. We observe violation of reflection positivity for the gluon propagator but not for the quark propagator.

function $S$ of Euclidean field theory has to fulfill the condition [45]

$$
\int_{0}^{\infty} d t d t^{\prime} \bar{f}\left(t^{\prime}, \vec{p}\right) S\left(-\left(t+t^{\prime}\right), \vec{p}\right) f(t, \vec{p})>0
$$

if a physical particle is described. Here $f$ are complex valued test functions. A violation of this condition signals the absence of the corresponding particle from the physical spectrum of the theory, i.e. the particle is confined. The one-dimensional Fourier transform $S(t, \vec{p})$ of the propagator $S\left(p_{0}, \vec{p}\right)$ is given by

$$
S(t, \vec{p}):=\int \frac{d p_{0}}{2 \pi} S\left(p_{0}, \vec{p}\right) e^{i p_{0} t}
$$

Provided there is a region around $t_{0}$ where $S\left(-t_{0}, \vec{p}\right)<0$ one can choose a real test function $f(t)$ which peaks strongly at $t_{0}$ to show positivity violation. In the following we chose $\vec{p}=0$. 
In the first diagram of Fig. 10] we display the Fourier transform of the nontrivial part $D\left(p^{2}\right)=Z\left(p^{2}\right) / p^{2}$ of the gluon propagator. Clearly one observes negative values on a large interval. The resulting positivity violation for the transverse gluon propagator in Landau gauge is a clear signal for gluon confinement. This corroborates previous findings in the quenched approximation [5]. These positivity violations have also been observed in lattice studies, see ref. [12] for recent corresponding results or ref. [46] for a review.

In the quark propagator positivity violations have been found in model studies, which solve the quark Dyson-Schwinger equation with an ansatz for the gluon propagator as input (see [2, 47, 48] and references therein). Similar violations have been found in $(2+1)$ dimensional QED [49]. For the model of ref. [33] or a propagator with complex conjugate poles [50] we tested our numerical routines and found positivity violations very easily. Contrary to these findings we do not observe positivity violations for the quark propagator from the coupled set of DSEs. The lower panel of Fig. 10] shows our results for the Fourier transform of the vector part $\sigma_{V}\left(p^{2}\right)=A\left(p^{2}\right) /\left(p^{2} A\left(p^{2}\right)+B\left(p^{2}\right)\right)$ and the scalar part $\sigma_{S}\left(p^{2}\right)=A\left(p^{2}\right) /\left(p^{2} A\left(p^{2}\right)+B\left(p^{2}\right)\right)$ of our solutions for the quark propagator employing four different quark-gluon vertices. All our solutions appear to be positive definite at the present level of numerical accuray. However, a more accurate study is required to settle this point 51]. Furthermore note that even if confirmed our findings are not in contradiction with the absence of quarks from the physical spectrum of QCD as violation of positivity is a sufficient but not a necessary condition for confinement.

\section{SUMMARY}

We have presented solutions of the (truncated) Dyson-Schwinger equations for the propagators of Landau gauge QCD. We first concentrated on the Dyson-Schwinger equation for the quark propagator. We proposed several ansätze for the quark-gluon vertex which consist of an Abelian part carrying the tensor structure of the vertex and a non-Abelian multiplicative correction. Our guiding principles for the construction of these vertices have been two important conditions on the truncated quark equation: it should be multiplicatively renormalizable and recover perturbation theory for large external momenta. In our truncation scheme the quark mass function is, as required from general arguments, independent of the renormalization point and has the correct asymptotic behavior for large momenta.

In the quark equation both the ghost and gluon dressing function show up at least implicitly. In quenched approximation, which is suitable to compare to lattice results, we employ solutions of the ghost and gluon Dyson-Schwinger equations taken from ref. 44]. In a second step we included the back-reaction of the quarks on the ghost and gluon system and solved the quark, gluon and ghost Dyson-Schwinger equations self-consistently.

All our solutions exhibit dynamical chiral symmetry breaking. However, only carefully constructed vertex ansätze have been able to generate masses in the typical phenomenological range of $300-400 \mathrm{MeV}$. Constructions with an Abelian part satisfying the Abelian Ward-identity are superior to other vertex ansätze. We obtained very good results for the quark mass, the pion decay constant and the chiral condensate by employing a generalized Curtis-Pennington [23] vertex. In the chiral limit both, the quark mass function and the vector self energy coincide with recently obtained lattice results [13, 14] within the numerical uncertainty. This agreement confirms the quality of our truncation and in turn shows that chiral extrapolation on the lattice works well.

In the unquenched case including the quark-loop in the gluon equation with $N_{f}=3$ 
light quarks we obtain only small corrections compared to the quenched calculations. In particular for the case of dynamically generated quark masses the quark-loop turns out to be suppressed in the gluon equation for small momenta. We thus showed on the level of our truncation that the Kugo-Ojima confinement criterion [38, 39] and Zwanziger's horizon condition [8, 40] are satisfied in Landau gauge QCD.

Furthermore we searched for positivity violations in the gluon and quark propagators. We confirmed previous findings [5] that the gluon propagator shows violation of reflection positivity. Thus the gluon is not contained in the physical state space of QCD. We did not find similar violations for the quark propagator. This issue is currently investigated in a more detailed study [51].

\section{Acknowledgments}

We are indebted to $\mathrm{P}$. Maris and $\mathrm{P}$. Watson for a critical reading of the manuscript and useful comments. We are grateful to P. Bowman for communicating lattice data. We thank S. Ahlig, J. Bloch, K. Langfeld, H. Reinhardt, C. Roberts, L. von Smekal, P. Tandy and A. Williams for helpful discussions.

This work has been supported by the DAAD and the DFG under contracts Al 279/3-4 and GRK683 (European graduate school Tübingen-Basel).

\section{APPENDIX A: ANGULAR INTEGRALS}

The angular integrals employed in the ultraviolet analysis are given by

$$
\begin{aligned}
\int_{0}^{\pi} d \theta \frac{\sin ^{2}(\theta)}{z^{2}} & =\frac{\pi}{2}\left[\frac{\Theta(x-y)}{x(x-y)}+\frac{\Theta(y-x)}{y(y-x)}\right] \\
\int_{0}^{\pi} d \theta \frac{\sin ^{2}(\theta)}{z} & =\frac{\pi}{2}\left[\frac{\Theta(x-y)}{x}+\frac{\Theta(y-x)}{y}\right] \\
\int_{0}^{\pi} d \theta \sin ^{2}(\theta) & =\frac{\pi}{2} \\
\int_{0}^{\pi} d \theta \sin ^{2}(\theta) z & =\frac{\pi}{2}(x+y) \\
\int_{0}^{\pi} d \theta \sin ^{2}(\theta) z^{2} & =\frac{\pi}{2}\left((x+y)^{2}+x y\right)
\end{aligned}
$$

where the squared momentum $z$ is defined as $z=(p-q)^{2}=x+y-2 \sqrt{x y} \cos (\theta)$.

[1] R. Alkofer and L. von Smekal, Phys. Rept. 353, 281 (2001) arXiv:hep-ph/0007355.

[2] C. D. Roberts and S. M. Schmidt, Prog. Part. Nucl. Phys. 45, S1 (2000) arXiv:nucl-th/0005064. 
[3] M. R. Pennington, in Proceedings of the Workshop on Nonperturbative Methods in Quantum Field Theory, edited by A. W. Schreiber, A. G. Williams, and A. W. Thomas, p. 49, Adelaide, 1998, World Scientific; arXiv:hep-th/9806200.

[4] C. S. Fischer and R. Alkofer, Phys. Lett. B536, 177 (2002) arXiv:hep-ph/0202202; C. S. Fischer, R. Alkofer and H. Reinhardt, Phys. Rev. D65, 0940082002 arXiv:hep-ph/0202195; R. Alkofer, C. S. Fischer and L. von Smekal, Acta Phys. Slov. 52, 191 (2002) arXiv:hep-ph/0205125.

[5] L. von Smekal, R. Alkofer and A. Hauck, Phys. Rev. Lett. 79, 3591 (1997) arXiv:hep-ph/9705242; L. von Smekal, A. Hauck and R. Alkofer, Annals Phys. 267, 1 (1998) arXiv:hep-ph/9707327; A. Hauck, L. von Smekal and R. Alkofer, Comput. Phys. Commun. 112 (1998) 166 arXiv:hep-ph/9804376.

[6] D. Atkinson and J. C. Bloch, Phys. Rev. D58, 094036 (1998) arXiv:hep-ph/9712459, D. Atkinson and J. C. Bloch, Mod. Phys. Lett. A 13, 1055 (1998) arXiv:hep-ph/9802239.

[7] C. Lerche and L. von Smekal, Phys. Rev. D 65, 125006 (2002) arXiv:hep-ph/0202194.

[8] D. Zwanziger, Phys. Rev. D 65, 094039 (2002) arXiv:hep-th/0109224.

[9] H. Suman and K. Schilling, Phys. Lett. B373 (1996) 314-318, arXiv:hep-lat/9512003.

[10] F. D. Bonnet et al., Phys. Rev. D 62, 051501 (2000) arXiv:hep-lat/0002020; Phys. Rev. D 64, 034501 (2001) arXiv:hep-lat/0101013.

[11] A. Cucchieri, Phys. Lett. B422 (1998) 233-237, arXiv:hep-lat/9709015; A. Cucchieri and D. Zwanziger, Phys. Lett. B524 (2002) 123-128, arXiv:hep-lat/0012024.

[12] K. Langfeld, H. Reinhardt and J. Gattnar, Nucl. Phys. B 621, 131 (2002) arXiv:hep-ph/0107141; Nucl. Phys. Proc. Suppl. 106, 673 (2002) arXiv:hep-lat/0110025; K. Langfeldet al., arXiv:hep-th/0209173.

[13] F. D. Bonnet, P. O. Bowman, D. B. Leinweber, A. G. Williams and J. B. Zhang [CSSM Lattice collaboration], Phys. Rev. D 65, 114503 (2002) arXiv:hep-lat/0202003 . J. B. Zhang, F. D. Bonnet, P. O. Bowman, D. B. Leinweber and A. G. Williams, arXiv:hep-lat/0208037.

[14] P. O. Bowman, U. M. Heller, D. B. Leinweber and A. G. Williams, arXiv:hep-lat/0209129.

[15] J. C. Taylor, Nucl. Phys. B 33 (1971) 436.

[16] W. J. Marciano and H. Pagels, Phys. Rept. 36, 137 (1978).

[17] P. C. Tandy, arXiv:nucl-th/0301040.

[18] J. C. Bloch, Phys. Rev. D 66, 034032 (2002) arXiv:hep-ph/0202073.

[19] J. Skullerud and A. Kizilersu, JHEP 0209, 013 (2002) arXiv:hep-ph/0205318.

[20] L. von Smekal, Habilitationsschrift, Friedrich-Alexander Universität, Erlangen-Nürnberg (1998).

[21] S. Ahlig (2001), Ph.D. thesis, Universität Tübingen, url: http://w210.ub.uni-tuebingen.de/dbt/volltexte/2001/280

[22] J. S. Ball and T. W. Chiu, Phys. Rev. D 22, 2542 (1980).

[23] D. C. Curtis and M. R. Pennington, Phys. Rev. D 42, 4165 (1990).

[24] V. A. Miransky, Phys. Lett. B 165, 401 (1985).

[25] R. Alkofer, C. S. Fischer and L. von Smekal, arXiv:hep-ph/0209366.

[26] D. Becirevic, P. Boucaud, J. P. Leroy, J. Micheli, O. Pene, J. Rodriguez-Quintero and C. Roiesnel, Phys. Rev. D 61 (2000) 114508 arXiv:hep-ph/9910204.

[27] K. Langfeld, R. Alkofer and P. A. Amundsen, Z. Phys. C 42, 159 (1989).

[28] P. Maris, C. D. Roberts and P. C. Tandy, Phys. Lett. B 420, 267 (1998) arXiv:nucl-th/9707003.

[29] A. Bender, W. Detmold, C. D. Roberts and A. W. Thomas, Phys. Rev. C 65, 065203 (2002) 
arXiv:nucl-th/0202082; A. Bender, C. D. Roberts and L. Von Smekal, Phys. Lett. B 380, 7 (1996) arXiv:nucl-th/9602012; G. Hellstern, R. Alkofer and H. Reinhardt, Nucl. Phys. A 625, 697 (1997) arXiv:hep-ph/9706551.

[30] H. Pagels and S. Stokar, Phys. Rev. D 20, 2947 (1979).

[31] C. D. Roberts and A. G. Williams, Prog. Part. Nucl. Phys. 33, 477 (1994) arXiv:hep-ph/9403224.

[32] P. Maris and C. D. Roberts, Phys. Rev. C 56, 3369 (1997) arXiv:nucl-th/9708029.

[33] R. Alkofer, P. Watson and H. Weigel, Phys. Rev. D 65, 094026 (2002) arXiv:hep-ph/0202053.

[34] K. Hagiwara et al. [Particle Data Group Collaboration], Phys. Rev. D 66, 010001 (2002).

[35] A. Kizilersu, A. W. Schreiber and A. G. Williams, Phys. Lett. B 499, 261 (2001) arXiv:hep-th/0010161.

[36] N. Brown and M. R. Pennington, Phys. Rev. D 38, 2266 (1988).

[37] J. C. Bloch, Ph.D. thesis - University of Durham (1995) arXiv:hep-ph/0208074.

[38] T. Kugo and I. Ojima, Prog. Theor. Phys. Suppl. 66 (1979) 1.

[39] T. Kugo, Int. Symp. on BRS symmetry, Kyoto, Sep. 18-22, 1995, arXiv:hep-th/9511033.

[40] D. Zwanziger, Nucl. Phys. B378 (1992) 525-590.

[41] B. V. Geshkenbein, arXiv:hep-ph/0206094.

[42] C. S. Fischer (2003), Ph.D. thesis, Universität Tübingen.

[43] S. Aoki, Nucl. Phys. Proc. Suppl. 94 (2001) 3-18, arXiv:hep-lat/0011074.

[44] R. Alkofer, A. Bender and C. D. Roberts, Int. J. Mod. Phys. A 10 (1995) 3319 arXiv:hep-ph/9312243.

[45] R. Haag, "Local quantum physics: Fields, particles, algebras,". Berlin, Germany: Springer (1992) 356 p. (Texts and monographs in physics).

[46] J. E. Mandula, Phys. Rept. 315 (1999) 273.

[47] A. Bender, D. Blaschke, Y. Kalinovsky and C. D. Roberts, Phys. Rev. Lett. 77 (1996) 3724 arXiv:nucl-th/9606006.

[48] A. Bender, G. I. Poulis, C. D. Roberts, S. M. Schmidt and A. W. Thomas, Phys. Lett. B 431 (1998) 263 arXiv:nucl-th/9710069.

[49] P. Maris, Phys. Rev. D 52 (1995) 6087 arXiv:hep-ph/9508323.

[50] M. Stingl, Z. Phys. A353 (1996) 423-445, arXiv:hep-th/9502157.

[51] R. Alkofer, W. Detmold, C. S. Fischer and P. Maris, in preparation.

[52] For a discussion of the relation of a $\widetilde{M O M}$-scheme to the $\overline{M S}$-scheme see section IV of ref. [26]. Based on a three loop calculation the authors obtained the relation $\Lambda_{\overline{M S}} \simeq 0.346 \Lambda_{\widetilde{M O M}}$. In our case this would result in the value $\Lambda_{\overline{M S}}=0.246$ which for zero flavors is even on the low side.

[53] Graphs for the choices $d=\delta$ and $d=0.1$ can be found in ref. [42]. 